\title{
Investigation of Structure-Activity Relationships of Dexrazoxane Analogs Reveals Topoisomerase II $\beta$ Interaction as a Prerequisite for Effective Protection against Anthracycline Cardiotoxicity $\$$
}

\author{
D Petra Kollárová-Brázdová, (D)Anna Jirkovská, (1)Galina Karabanovich, (DZuzana Pokorná, \\ (D) Hana Bavlovič Piskáčková, (D) Eduard Jirkovský, (DJan Kubeš, (DOlga Lenčová-Popelová, \\ (1) Yvona Mazurová, (D) Michaela Adamcová, (DVeronika Skalická, (D)Petra Štěrbová-Kovaříková, \\ (D) Jaroslav Roh, (1)Tomáš Šimůnek, and (1) Martin Štěrba
}

Departments of Pharmacology (P.K.-B., Z.P., E.J., O.L.-P., M.S.), Histology and Embryology (Y.M.), and Physiology (M.A.), Faculty of Medicine in Hradec Králové, Charles University, Hradec Králové, Czech Republic; and Departments of Biochemical Sciences (A.J., J.K., V.S., T.S.), Organic and Bioorganic Chemistry (G.K., J.R.), Pharmaceutical Chemistry and Pharmaceutical Analysis (H.B.P., P.S.-K.), and Pharmacology and Toxicology (E.J.), Faculty of Pharmacy in Hradec Králové, Charles University, Hradec Králové, Czech Republic

Received December 19, 2019; accepted March 23, 2020

\begin{abstract}
Bisdioxopiperazine agent dexrazoxane (ICRF-187) has been the only effective and approved drug for prevention of chronic anthracycline cardiotoxicity. However, the structure-activity relationships (SARs) of its cardioprotective effects remain obscure owing to limited investigation of its derivatives/analogs and uncertainties about its mechanism of action. To fill these knowledge gaps, we tested the hypothesis that dexrazoxane derivatives exert cardioprotection via metal chelation and/or modulation of topoisomerase $\| \beta$ (Top2B) activity in chronic anthracycline cardiotoxicity. Dexrazoxane was alkylated in positions that should not interfere with the metal-chelating mechanism of cardioprotective action; that is, on dioxopiperazine imides or directly on the dioxopiperazine ring. The protective effects of these agents were assessed in vitro in neonatal cardiomyocytes. All studied modifications of dexrazoxane molecule, including simple methylation, were found to abolish the cardioprotective effects. Because this challenged the prevailing mechanistic concept and previously reported data, the two closest derivatives [( \pm )-4,4'-(propane-1,2-diyl)bis(1-methylpiperazine-2,6-dione) and 4-(2-(3,5-dioxopiperazin-1-yl)ethyl)-3methylpiperazine-2,6-dione] were thoroughly scrutinized in vivo using a rabbit model of chronic anthracycline cardiotoxicity. In
\end{abstract}

contrast to dexrazoxane, both compounds failed to protect the heart, as demonstrated by mortality, cardiac dysfunction, and myocardial damage parameters, although the pharmacokinetics and metal-chelating properties of their metabolites were comparable to those of dexrazoxane. The loss of cardiac protection was shown to correlate with their abated potential to inhibit and deplete Top2B both in vitro and in vivo. These findings suggest a very tight SAR between bisdioxopiperazine derivatives and their cardioprotective effects and support Top2B as a pivotal upstream druggable target for effective cardioprotection against anthracycline cardiotoxicity.

\section{SIGNIFICANCE STATEMENT}

This study has revealed the previously unexpected tight structure-activity relationships of cardioprotective effects in derivatives of dexrazoxane, which is the only drug approved for the prevention of cardiomyopathy and heart failure induced by anthracycline anticancer drugs. The data presented in this study also strongly argue against the importance of metalchelating mechanisms for the induction of this effect and support the viability of topoisomerase $\| \beta$ as an upstream druggable target for effective and clinically translatable cardioprotection.

\section{Introduction}

Anthracyclines (ANTs), such as doxorubicin or daunorubicin (DAU), rank among the most effective anticancer drugs. However, their clinical use is hampered by the occurrence of cardiotoxicity. In particular, the chronic forms, which are

This study was supported by the Charles University Grant Agency (GAUK) 680216; Czech Science Foundation (GACR) 18-08169S; Charles University Research Program Progres [Grant Q40/5]; and by project Pre-application research into innovative medicines and medical technologies (InoMed) reg. No. CZ.02.1.01/0.0/0.0/18_069/0010046 co-funded by the European Union.

Conflict of interest: None declared.

https://doi.org/10.1124/jpet.119.264580.

S This article has supplemental material available at jpet.aspetjournals.org. largely irreversible and manifest months or years after cancer chemotherapy (usually as a dilated cardiomyopathy and congestive heart failure), are of concern (Bloom et al., 2016). Besides the aforementioned issues in human medicine, this toxicity also complicates oncological treatments in veterinary medicine. The mechanisms of ANT cardiotoxicity have not yet been fully elucidated. The main role has been attributed to oxidative damage to the myocardium induced by the redox cycling of ANT molecules with the catalytic involvement of free iron (Hasinoff et al., 1998; Simůnek et al., 2009). However, antioxidants and selective iron-chelators have been unable to provide robust cardioprotection when examined in clinical studies or relevant experimental models of chronic ANT 
cardiotoxicity (Stěrba et al., 2013). Recently, topoisomerase II $\beta$ (Top2B) interaction in the heart has been proposed as the pivotal event for ANT cardiotoxicity development (Zhang et al., 2012). This challenges not only the original mechanistic concept but also traditional routes for the development of effective cardioprotection.

Dexrazoxane (DEX) (ICRF-187) is the only cardioprotective drug that has been approved for the prevention of ANT cardiotoxicity in clinical practice (Reichardt et al., 2018). Its cardioprotective effects have been well-established in experimental settings (Jirkovský et al., 2013; Herman et al., 2014) as well as in multiple randomized clinical trials (van Dalen et al., 2011). Although DEX is unambiguously an effective cardioprotectant, its current clinical use is limited. Early concerns regarding its possible impact on cancer response (Swain et al., 1997) resulted in the restriction of DEX use only to patients exceeding a cumulative dose of $300 \mathrm{mg} / \mathrm{m}^{2}$ of doxorubicin (https://www.ema.europa.eu/en/documents/referral/ dexrazoxane-h-31-1275-article-1231-referral-assessmentreport_en.pdf). Later, concerns about the increased risk of secondary malignancies in pediatrics (Tebbi et al., 2007) resulted in the temporary contraindication of DEX in children (https:// www.ema.europa.eu/en/documents/referral/dexrazoxane-h-311275-article-1231-referral-assessment-report_en.pdf). However, the vast majority of recent data on DEX in pediatric and adult patients did not support these claims, and a revocation of the contraindication in children followed (van Dalen et al., 2011; Reichardt et al., 2018; Kim et al., 2019).

DEX has been traditionally considered as a prodrug of the metal-chelating agent ADR-925, which should prevent ANTinduced oxidative stress and myocardial damage by sequestration of labile (redox-active) iron ions with the subsequent prevention of ROS formation (Hasinoff, 1989; Simůnek et al., 2009). DEX has also been shown to interact with Top2B, and this was linked to the induction of cardioprotection (Lyu et al., 2007; Lenčová-Popelová et al., 2016); however, it remains unclear whether Top2B interaction is indispensable for cardioprotection or a dispensable part of a complex mechanism of action.

Bisdioxopiperazine agents, such as razoxane (ICRF-159, a racemic form of DEX), were originally developed and studied as anticancer agents (Creighton et al., 1969; Hasinoff et al., 1995). Only a few studies have investigated the cardioprotective effects of bisdioxopiperazine derivatives other than DEX (Herman et al., 1997; Martin et al., 2009; Jirkovská-Vávrová et al., 2015; Bures et al., 2017). Correspondingly, structureactivity relationships (SARs) in these settings remain poorly characterized. Herman et al. (1997) reported that the functionalization of DEX imides, which does not preclude the hydrolytic opening and metal-chelating qualities of the metabolite, retains cardioprotective potential against ANT cardiotoxicity in vivo. This concerned agents hydrolysable in a biologic environment to razoxane (Liang et al., 1999) but also derivatives with permanent alkylation on the imides. The latter is exemplified by the $N, N$-dimethyl derivative of DEX (ICRF-239), which has been reported to have significant cardioprotective effects against chronic ANT cardiotoxicity, as judged by the myocardial histopathology (Herman et al., 1997). This observation could open yet unexploited routes toward new DEX derivatives with potentially improved pharmaceutical and pharmacological properties. The current literature also does not suggest whether functionalization of the dioxopiperazine ring in position 3 may have a positive impact on cardioprotection.

This study aimed to investigate the in vitro and in vivo cardioprotective effects of two series of DEX derivatives to elucidate the SAR with respect to the cardioprotective effects. We also tested the hypothesis that DEX derivatives exert cardioprotection via metal chelation and/or modulation of Top2B activity in chronic ANT cardiotoxicity. The first studied series of compounds stemmed from the alkylation of both dioxopiperazine imides, whereas the second was based on the functionalization of one 2,6-dioxopiperazine ring in position 3 with short alkyl. Pharmacokinetics and key pharmacodynamic aspects (metal chelation and Top2B interaction) were examined and compared with DEX to explain the observed effects.

\section{Materials and Methods}

\section{Materials}

Dulbecco's modified Eagle's medium: nutrient mixture F-12 (DMEM/F12), horse serum, FBS, penicillin/streptomycin solution $(5000 \mathrm{U} / \mathrm{ml})$, and sodium pyruvate solution $(100 \mathrm{mM})$ were purchased from Lonza (Basel, Switzerland). The sera were heat-inactivated prior to use. RPMI-1640 medium with L-glutamine and $\mathrm{NaHCO}_{3}$, lactic acid, nicotinamide adenine dinucleotide, 3-(4,5-dimethylthiazol-2-yl)2,5-diphenyltetrazolium bromide, DMSO, and other chemicals (e.g., constituents of various buffers and chemicals for LC-MS analysis) were purchased from Sigma-Aldrich (St. Louis, MO) or Penta (Prague, Czech Republic) and were of the highest available pharmaceutical or analytical grade unless stated otherwise.

Substances of DAU and DEX were purchased as hydrochloric salts (pharmaceutical grade) from Euroasian Chemicals Pvt. Ltd. (Mumbai, India). The identity and purity of the substances were confirmed inhouse using high performance liquid chromatography-mass spectrometry (HPLC-MS). DEX derivatives/analogs were synthetized and characterized as described below. All other chemicals were purchased from Sigma-Aldrich unless stated otherwise.

\section{Synthesis of DEX Derivatives}

Details of the synthesis of DEX derivatives and their corresponding identification data can be found in the Supplemental Materials and Methods.

Series of racemic $N, N$-dialkyl DEX derivatives were prepared inhouse from razoxane by reactions with a corresponding aliphatic alcohol using the protocol of the Mitsunobu reaction (Swamy et al., 2009). Dimethyl, diethyl, diisopropyl, and dibenzyl derivatives were prepared and labeled as ( \pm )-4,4'-(propane-1,2-diyl)bis(1-methylpiperazine-2,6-dione) (GK-627), GK-638, GK-639, and GK-635, respectively 
<smiles>CC(CN1CC(=O)NC(=O)C1)N1CC(=O)NC(=O)C1</smiles>

DEX<smiles>[R]N1C(=O)CN(CC(C)N2CC(=O)N([R])C(=O)C2)CC1=O</smiles>

$\mathrm{R}=\mathrm{Me}: \quad \mathrm{GK}-627$

$\mathrm{R}=\mathrm{Et}: \quad$ GK-638

$\mathrm{R}=i \mathrm{Pr}: \quad$ GK-639

$\mathrm{R}=\mathrm{Bn} \quad$ GK-635

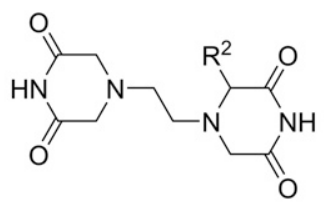

$\mathrm{R}^{2}=$ Me: GK-580

$\mathrm{R}^{2}=\mathrm{Et}: \quad$ GK-5954<smiles>CC(CN(CC(N)=O)CC(=O)O)N(CC(N)=O)CC(=O)O</smiles>

ADR-925

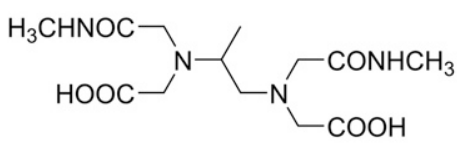

GK-627 met<smiles>CC(C(N)=O)N(CCN(CC(N)=O)CC(=O)O)CC(=O)O</smiles>

GK-580 ${ }_{\text {met }}$

Fig. 1. Chemical structures of DEX, its derivatives, and their ring-opened metabolites. The first group of DEX derivatives was substituted on imide nitrogen with methyl (Me) (GK-627), ethyl (Et) (GK-638), isopropyl $(i \mathrm{Pr})$ (GK-639), or benzyl (Bn) (GK-635) substituents. The second group included derivative GK-580, in which the methyl group was moved from the aliphatic linker to position 3 in one 2,6-dioxopiperazine ring, and its ethyl analog (GK-5954). The corresponding ring-opened metabolites (met) of DEX (ADR-925), GK-627 (GK-627 met), and GK-580 (GK-580 ${ }_{\text {met }}$ ) are displayed under the line.

(Fig. 1). This approach was employed for the synthesis of the studied derivatives because of its capability to prepare all four derivatives under mild reaction conditions with satisfactory yields. This was in contrast to two alternative synthetic approaches tested for this purpose: 1) the synthesis of compound GK-627 using $N$-methylformamide, as described previously (Creighton, 1992; Hellmann, 1993), was found to be unsuitable for the synthesis of other $N, N$-dialkyl derivatives; and 2) the alkylation of razoxane with dimethyl sulfate in the presence of a base provided GK-627 only in low yields, whereas the alkylation of razoxane with alkyl halides did not provide some of the other derivatives at all.

Compound 4-(2-(3,5-dioxopiperazin-1-yl)ethyl)-3-methylpiperazine2,6-dione (GK-580) and its ethyl derivative GK-5954 (Fig. 1) were prepared using a five-step procedure beginning with commercially available $N$-benzylethylenediamine. In the final step, the corresponding tetracarboxylic acids in formamide were cyclized according to the standard protocol of DEX synthesis (Creighton, 1976), providing good yields of methyl (GK-580) and ethyl (GK-5954) derivatives of DEX.

\section{In Vitro Experiments}

Assessment of the Protective Effects of DEX and Its Derivatives against ANT Toxicity. The protective effects of DEX and its derivatives against ANT-induced cytotoxicity have been evaluated using a well-established in vitro model utilizing primary neonatal cardiomyocytes (Vavrova et al., 2013; Jirkovská-Vávrová et al., 2015). Primary neonatal ventricular rat cardiomyocytes (NVCMs)

were isolated from 1- to 3-day-old Wistar rats (sex undetermined, presumably mixed; Velaz, Prague, Czech Republic) as described previously (Vavrova et al., 2013). Animal use was approved by the Animal Welfare Committee of the Faculty of Pharmacy in Hradec Králové, Charles University.

After isolation and myocyte separation, the suspension of NVCMs was plated on 24 -well plates precoated with $1 \%$ gelatin at a density of $0.4 \times 10^{6}$ cells per well. NVCMs were cultured at $37^{\circ} \mathrm{C}$ and $5 \% \mathrm{CO}_{2}$ in DMEM/F12 supplemented with $10 \%$ horse serum, $5 \%$ FBS, $4 \%$ sodium pyruvate solution, and $1 \%$ penicillin/streptomycin solution. Newly isolated NVCMs were left for 40 hours to attach, and the medium was changed to DMEM/F12 supplemented with 5\% FBS, $4 \%$ sodium pyruvate solution, and $1 \%$ penicillin/streptomycin solution. After another 24 hours, the medium was replaced once more. All experiments were initiated on the 4 th day after isolation, when the confluent cellular monolayer of synchronically beating cardiomyocytes was obtained. Serum- and pyruvate-free media were used for the experiments.

ANT toxicity was induced with clinically relevant concentration of DAU $(1.2 \mu \mathrm{M})$ (Paul et al., 1989; Varatharajan et al., 2016). The protective effects of DEX and its derivatives were tested in a clinically relevant range of concentrations $(10-100 \mu \mathrm{M})$. The cells were preincubated with DEX or its derivatives for 3 hours at $37^{\circ} \mathrm{C}$ and then coincubated with $1.2 \mu \mathrm{M}$ DAU for another 3 hours. Thereafter, the medium was changed, and drug-free postincubation followed for 48 hours. Cytotoxicity was evaluated using a lactate dehydrogenase (LDH) assay, as described previously (Vavrova et al., 2013). The results were expressed as the percentage of total cellular LDH measured after lysis of the cells in lysis buffer. The toxicity of DEX and its derivatives were assayed by continual incubation with the given concentration of the single agent for 48 hours at $37^{\circ} \mathrm{C}$. Drugs were dissolved in DMSO, and the final concentration of DMSO in the cell experiments (including control cells) was $0.1 \%$. Four to six independent experiments were performed in each group.

Antiproliferative Study. The effect of the agents on the proliferation of cancer cells was studied using the HL-60 cell line (lot 5036502; American Type Culture Collection, Manassas, VA) derived from patient with acute promyelocytic leukemia. The cells were cultured in RPMI-1640 medium supplemented with 10\% FBS and $1 \%$ penicillin/streptomycin solution in $75-\mathrm{cm}^{2}$ tissue culture flasks at $37^{\circ} \mathrm{C}$ in a humidified atmosphere of $5 \% \mathrm{CO}_{2}$. To assess the impact of the agents on proliferation, HL-60 cells were plated on 96 -well plates (10,000 cells per well) and incubated with DAU (1-300 nM) and/or DEX/DEX derivatives $(1-100 \mu \mathrm{M})$ for 72 hours at $37^{\circ} \mathrm{C}$. The rate of proliferation was determined using the 3-(4,5-dimethylthiazol-2-yl)2,5-diphenyltetrazolium bromide assay, as described previously (Vavrova et al., 2013), and expressed as a percentage of control (untreated) cells. The drugs were dissolved in DMSO, and the final concentration of DMSO in cell experiments (including control cells) was $0.1 \%$. In each group, five independent experiments were performed.

\section{In Vivo Cardioprotective Study}

The cardioprotective potential of selected agents was also studied in vivo using well-established and DEX-validated model of chronic ANT cardiotoxicity in male rabbits using $3 \mathrm{mg} / \mathrm{kg}$ DAU per week for 10 consecutive weeks (Simůnek et al., 2004; Popelová et al., 2009; Jirkovský et al., 2013). The individual dose of DAU used in this study corresponds to approximately $50 \mathrm{mg} / \mathrm{m}^{2}$, which is the dose used in human clinical practice (EMC, 2019). All animal procedures were approved and supervised by the Animal Welfare Committee of the Faculty of Medicine in Hradec Králové, Charles University.

New Zealand White male rabbits $(3.3 \pm 0.3 \mathrm{~kg}, 12-16$ weeks old, $n=$ 54 ; Velaz) were randomly allocated to seven groups and treated weekly for 10 consecutive weeks. The choice of sex corresponded with the well-established model and aimed for a high reproducibility of results (e.g., by avoiding variations due to estrus). Cardiotoxicity was induced by DAU ( $3 \mathrm{mg} / \mathrm{kg}$, i.v.; DAU group, $n=10$ ), whereas the control 
group received saline $[1 \mathrm{ml} / \mathrm{kg}$, i.v.; control (CTR) group, $n=8]$. The cardioprotective drug DEX was used as a positive control at the previously established and clinically recommended ratio to ANT of 20: 1 (60 mg/kg, i.p., 30 minutes before each DAU; DEX + DAU group, $n=$ 7). Of all the prepared DEX derivatives, the two with the smallest changes in their chemical structure (GK-627 and GK-580) were selected for the in vivo study. They were administered at the same dose $(60 \mathrm{mg} / \mathrm{kg}$, i.p.) and schedule (30 minutes before each DAU) as DEX (GK-627 + DAU and GK-580 + DAU groups, $n=8$ in each group). GK-627 and GK-580 were also administered individually in the same manner ( $n=8$ and $n=5$, respectively). Animal group sizes were designed with respect to previous experience with this model (e.g., typical mortality) and pre hoc statistical power analysis (alpha $=0.05$, power > 0.8; SigmaStat 3.5 software; SPSS, Chicago, IL). All solutions were freshly prepared before administration. DEX and both derivatives were used as dihydrochlorides, and substances were completely dissolved. Final solutions $(60 \mathrm{mg} / \mathrm{ml})$ were filtered through antimicrobial $0.22-\mu \mathrm{M}$ porosity filters (Carl Roth GmbH + Co. KG, Karlsruhe, Germany) before administration.

Animals were caged individually under standard conditions (temperature $18^{\circ} \mathrm{C}$, relative humidity $40 \%-50 \%$, 12-hour illumination period) with free access to a standard rabbit chow diet (KO-16; Velas, a.s., Lysá and Labem, Czech Republic) and tap water. Body weight was monitored weekly, and behavior and mortality of the rabbits were monitored daily. Noninvasive measurements, drug administration, and blood sampling were performed under light anesthesia with ketamine (30 mg/kg, Narketan; Vétoquinol, Lure Cedex, Francie) and midazolam (1.25 mg/kg, Midazolam Torrex; Chiesi Pharmaceuticals, Vienna, Austria) administered in combination intramuscularly into the rabbit thigh. One week after the last dose of the drug, invasive hemodynamic measurement was performed under individually titrated pentobarbital anesthesia (3\% solution, i.v., $\approx 30 \mathrm{mg} / \mathrm{kg}$; SigmaAldrich). Pentobarbital was also used to euthanize the animals. During necropsy of animals (including for unscheduled deaths), peritoneal and pleural effusions were monitored. Subsequently, the heart was rapidly excised and briefly retrogradely perfused with icecold saline using a syringe. Transverse sections of cardiac ventricles were taken for histologic examination, and the rest of the free ventricles were shock frozen in liquid nitrogen, pulverized, and stored at $-80^{\circ} \mathrm{C}$.

Examination of Cardiac Function. The left ventricular (LV) systolic function was assessed by echocardiography (Vivid 4, 10-MHz probe; GE Healthcare, Chicago, IL) at the start of the experiment and weekly from weeks 8 to 11 of the experiment, when the DAU-induced decline in systolic function was typically observed (Popelová et al., 2009). Left parasternal long- and short-axis M-mode scanning was used to obtain LV end-systolic and end-diastolic diameters for the calculation of LV fractional shortening, as described previously (Jirkovský et al., 2013).

At the end of the study, LV invasive hemodynamic measurement was performed via the arteria carotis sinistra using a Mikro-Tip pressure catheter (2.3F; Millar instruments, Houston, TX) connected to a data acquisition system (Powerlab; ADInstruments Pty., Bella Vista, Australia). The first derivative of the LV pressure change in the isovolumic contraction (index $d P / d t_{\max }$ ) was calculated using LabChart 6 software (ADInstruments Pty). Data were obtained after the stabilization period ( $\approx 15$ minutes).

Plasma Troponin T Analysis. Blood samples $(\approx 1 \mathrm{ml})$ were taken from the central ear artery into heparin-containing tubes (BD Vacutainers; BD Biosciences, Plymouth, UK) before the 1st, 5th, 8 th, and 10th drug administrations and before the invasive procedures at the end of the study. Cardiac troponin $\mathrm{T}(\mathrm{cTnT})$ plasma concentrations were determined using the Elecsys Troponin T-high sensitive STAT test (Roche Diagnostics, Basel, Switzerland) with a detection limit of $0.003 \mu \mathrm{g} / \mathrm{l}$.

Histopathological Examination. Transverse sections of the heart ventricles (about $5 \mathrm{~mm}$-thick) were immersed in $4 \%$ neutral formaldehyde for 5-7 days and embedded in paraffin. Serial paraffin sections ( $5 \mu \mathrm{m}$-thick) were stained with Masson's blue trichrome (used mainly to distinguish collagen fibers [dark blue-stained] and muscle tissue [red-stained]). Photomicrographs were made using an Olympus BX51 microscope equipped with a DP71 digital camera (Olympus, Tokyo, Japan) and QuickPHOTO 3.0 software (PROMICRA, Prague, Czech Republic).

\section{RNA Isolation and Quantitative Real-Time Polymerase Chain Reaction}

Pulverized samples of LV were used to isolate total RNA with TRI Reagent (Sigma-Aldrich). RNA was reverse transcribed to cDNA using the High-Capacity cDNA Reverse Transcription Kit, and quantitative real-time polymerase chain reaction (PCR) analysis was performed with a 7500HT Fast Real-Time PCR System using TaqMan Fast Universal PCR Master Mix (all from Applied Biosystems, Foster City, CA) and commercial quantitative PCR assays (Applied Biosystems or Generi Biotech, Hradec Králové, Czech Republic; for details, see Supplemental Materials and Methods). The expression of target genes was normalized to the reference gene hypoxanthine phosphoribosyltransferase 1 , and the relative gene expression was calculated using the Pfaffl method (Pfaffl, 2001).

\section{Pharmacokinetic Study in Rabbits}

New Zealand White male rabbits $(3.6 \pm 0.2 \mathrm{~kg}, 13-17$ weeks old, $n=$ 13; Velaz) were randomly allocated to three groups for the pilot pharmacokinetic experiments: DEX (60 mg/kg; $n=5)$, GK-627 (60 mg/kg; $n=4)$, and GK-580 (60 mg/kg; $n=4)$. The animals received the agents as a single intraperitoneal dose, as described in the cardioprotective study. Blood $(\approx 1.5 \mathrm{ml})$ was sampled from the central ear artery into heparin-containing tubes before drug administration and at predefined intervals (5 minutes to 12 hours) after drug administration. The blood was then immediately centrifuged $(3000 \mathrm{~g}$, 6 minutes), and plasma was collected, frozen in liquid nitrogen, and stored at $-80^{\circ} \mathrm{C}$ until analysis. Sterile saline was used to compensate for the blood volume of the rabbits. Midazolam, alone or in combination with ketamine, was used to maintain the sedation/light anesthesia necessary for blood sampling at later time intervals. Animals were euthanized 12 hours after drug administration by pentobarbital overdose. The above-described diet and housing conditions were also used in this part of the study.

The plasma samples taken from the pharmacokinetic study with DEX were analyzed using the HPLC-MS/MS assay described and validated previously (Kovarikova et al., 2013). Briefly, rabbit plasma $(100 \mu \mathrm{l})$ was spiked with internal standards, precipitated with ice-cold methanol $(600 \mu \mathrm{l})$, and vortexed (30 seconds). The samples were centrifuged ( 10 minutes, $4^{\circ} \mathrm{C}, 16,800 \mathrm{~g}$ ), and the resulting supernatant was filtered through a $0.45-\mu \mathrm{m}$ porosity filter (Milex-Hv; MerckMillipore, Darmstadt, Germany) and injected into the column. A Prominence LC-20A HPLC system (Shimadzu, Kyoto, Japan), coupled with an LCQ Advantage MAX mass spectrometer (Thermo Finnigan, San Jose, CA), was used for sample analysis. The analytes were ionized by electrospray in positive ion mode. The separation was performed on a Synergi Polar-RP column with a mixture of ammonium formate and methanol in a gradient mode as the mobile phase. This method was further adapted for the analysis of close DEX derivatives (GK-580 and GK-627) and their metabolites. GK-580 was analyzed using the same instrument, whereas the GK-627 assay was performed on a Nexera X2 UHPLC system coupled to an LCMS-8030 triple quadrupole mass spectrometer (both Shimadzu). Details of these methods can be found in the Supplemental Materials and Methods.

\section{Displacement of Iron Ions from Complex with Daunorubicin}

The spectrophotometric assay was adapted from Hasinoff et al., (2003). The DAU-Fe (3:1) complex solution was prepared by adding DAU solution in water to $\mathrm{FeCl}_{3}$ in $15 \mathrm{mM} \mathrm{HCl}$. The resulting reddishbrown mixture was added to the reaction buffer (50 mM Tris, $150 \mathrm{mM}$ 
$\mathrm{KCl}, \mathrm{pH}=7.4$, room temperature) to yield final concentrations of $45 \mu \mathrm{M}$ DAU and $15 \mu \mathrm{M} \mathrm{Fe}^{3+}$. The spectrophotometric measurement at $600 \mathrm{~nm}$ (absorption band typical for the complex compared with uncomplexed DAU) was performed in 96-well plates (eight parallel wells per measurement) using an Infinite M200 microplate reader (Tecan Group, Männedorf, Switzerland). After a 3-minute equilibration period, solutions of the tested compounds in DMSO $(100 \mu \mathrm{M}$ final concentration, $1 \% \mathrm{DMSO}$ ) or $1 \%$ DMSO alone (as a control) were added, and the absorbance at $600 \mathrm{~nm}$ was measured for another 10 minutes (600 seconds). Four to eight independent experiments were performed in each group.

\section{Inhibition of Topoisomerase II DNA Relaxation Activity}

The topoisomerase II activity assay was performed using recombinant human topoisomerase II $\alpha$ (Top2A) or Top2B, which were purified as described previously (Gilroy and Austin, 2011) and kindly provided by Prof. Austin. Either enzyme isoform was incubated with $1 \mathrm{nM}$ of supercoiled DNA plasmid TCS1 (Lee et al., 1989) in a reaction buffer containing $10 \mathrm{mM}$ Tris- $\mathrm{HCl}$ (pH 8.0), $50 \mathrm{mM} \mathrm{KCl}, 50 \mathrm{mM} \mathrm{NaCl}, 10 \mathrm{mM}$ $\mathrm{MgCl}_{2}, 0.1 \mathrm{mM}$ EDTA, $5 \mathrm{mM}$ ATP, $0.1 \mathrm{mg} / \mathrm{ml}$ bovine serum albumin, and $1 / 10$ volume of each compound diluted in $10 \%$ DMSO (final DMSO concentration $1 \%$ ) for 30 minutes at $37^{\circ} \mathrm{C}$. The reaction was then stopped by the addition of the gel loading buffer (1/10 volume; $1 \%$ SDS, $0.025 \%$ bromophenol blue, $20 \%$ sucrose, $5 \mathrm{mM}$ EDTA, $50 \mathrm{mM}$ TRIS$\mathrm{HCl}, \mathrm{pH}$ 8.0). The topological forms of the plasmid were resolved in a $1 \%$ agarose gel in Tris/borate/EDTA buffer ( $89 \mathrm{mM}$ Tris base, $89 \mathrm{mM}$ Boric acid, 2 mM EDTA, pH 8.3) at $3 \mathrm{~V} / \mathrm{cm}$ for approximately 2 hours. Gels were stained with ethidium bromide $(0.5 \mu \mathrm{g} / \mathrm{ml})$ for 15 minutes and visualized using the Gel Doc EZ system with ImageLab software (Bio-Rad, Hercules, CA).

\section{Western Blot Analysis of Top2B Protein Depletion}

Top2B Depletion in Samples from In Vitro Experiments in NVCMs. The NVCMs plated on 24 -well plates were incubated with $\mathrm{DEX}$ and all derivatives under study for 24 hours at $37^{\circ} \mathrm{C}$. Then, the cells were harvested by scraping in radioimmunoprecipitation assay buffer (Sigma-Aldrich) with phosphatase and protease inhibitor solutions (Thermo Fisher Scientific Inc., Waltham, MA and Roche, respectively) on ice. After centrifugation $\left(14,000 \mathrm{~g}, 4^{\circ} \mathrm{C}, 15\right.$ minutes $)$, the supernatant was collected, and $10 \mu \mathrm{g}$ of protein was loaded into each lane of a Mini-PROTEAN 7.5\% TGX Stain-Free FastCast Acrylamide gel (Bio-Rad). After separation, the proteins were transferred onto a $0.2-\mu \mathrm{m}$ nitrocellulose membrane using a Trans-Blot Turbo Transfer System (Bio-Rad). Rabbit monoclonal anti-Top2A/B antibody [EPR5377] (ab109524; dilution 1:1000; Abcam, Cambridge, UK) was used as the primary antibody and goat anti-rabbit IgG F(ab') horseradish peroxidase-conjugated antibody (ab6112; dilution 1: 10,000; Abcam) was used as the secondary antibody. Clarity Western ECL Substrate was used for detection on ChemiDoc with Image Laboratory software (all Bio-Rad). The signal was normalized to the total protein on the membrane visualized by Stain-Free technology.

Top2B Depletion in Samples from In Vivo Experiments. Samples of the LV myocardium obtained at the end of the pharmacokinetic experiments (12 hours after administration of a single dose of either DEX, GK-627, or GK-580 at $60 \mathrm{mg} / \mathrm{kg}$, i.p., similar to the cardioprotective study) were analyzed for their Top2B protein level. Rabbits treated intraperitoneally with saline $(1 \mathrm{ml} / \mathrm{kg}$, pH-adjusted; $n=5$ ) were used as appropriate controls. Pulverized LV samples were homogenized in ice-cold radioimmunoprecipitation assay buffer supplemented with protease (cOmplete, EDTA-free Protease Inhibitor Cocktail; Roche) and phosphatase inhibitors (Halt Phosphatase Inhibitor Cocktail; Thermo Fisher Scientific Inc.). Samples were processed to obtain equal amounts of proteins; the proteins were separated on precast gels (Mini-PROTEAN TGX 4\%-15\%; Bio-Rad) and transferred onto a PVDF membrane (Immobilon-P; SigmaAldrich) using a tank (wet) technique. The upper parts of the membranes were incubated with the same Top2B primary antibody as that used for NVCMs (ab109524; dilution 1:1000; Abcam) and secondary anti-rabbit antibody (NA934; dilution 1:2000; GE Healthcare, Buckinghamshire, UK). The signal was elicited using BM Chemiluminescence Western Blotting Substrate (Roche) and detected on CL-XPosure film (Thermo Fisher Scientific Inc.). The lower parts of the membranes were incubated with mouse anti-glyceraldehyde-3phosphate dehydrogenase primary antibody (G8795; dilution 1: 25,000; Sigma-Aldrich) and secondary anti-mouse antibody (Polyclonal Goat Anti-Mouse Immmunoglobulin/Horseradish Peroxidase; dilution 1:25,000; DAKODenmark A/S, Glostrup, Denmark) as a loading control. Quantity One software (Bio-Rad) was used for densitometric quantification of the bands.

\section{Data Analysis}

SigmaStat 3.5 software (SPSS) was used for statistical analyses. Statistical significance $(P<0.05)$ was determined by one-way or twoway ANOVA (followed by Holm-Sidak's post hoc test) or ANOVA on ranks (followed by Dunn's post hoc test) according to the data characteristics. Data are presented as mean \pm S.D. or as boxes and whiskers representing the interquartile range and 5 th-95th percentile, respectively, unless stated otherwise. GraphPad Prism 7.00 (GraphPad Software, San Diego, CA) was used for the graphical representation of the results.

\section{Results}

\section{Cytoprotective Effects of DEX and Its Derivatives against DAU Toxicity In Vitro}

Clinically relevant concentration of DAU $(1.2 \mu \mathrm{M})$ induced significant cytotoxicity as determined by $\mathrm{LDH}$ release in NVCMs (Fig. 2). DEX significantly protected the cells from the DAU toxicity at all tested concentrations $(10-100 \mu \mathrm{M})$ (Fig. 2A). In sharp contrast, none of the six close DEX derivatives elicited the same effect at concentrations up to $100 \mu \mathrm{M}$ (Fig. 2, B-G). Similar to DEX, most derivatives were nontoxic on their own. However, the dibenzyl derivative of DEX (GK-635) induced significant toxicity at the highest tested concentration $(100 \mu \mathrm{M})$ (Fig. $2 \mathrm{E}$ ). In combination experiments, it was observed that the toxicity of this compound apparently had an additive effect to that of DAU.

\section{In Vivo Cardioprotective Study}

The complete lack of in vitro cardioprotective effects of all DEX derivatives, including the closest derivatives (dimethyl derivative GK-627 and isomethyl derivative GK-580) was surprising. In a previous in vivo study, the former drug (also known as ICRF-239) was reported to decrease ANT-induced myocardial damage in a DEX-validated model of chronic ANT cardiotoxicity (Herman et al., 1997). Indeed, chronic toxicity cannot be completely mimicked in vitro. Discrepancy between the in vitro data obtained in NVCMs and in vivo data obtained on a model of chronic ANT cardiotoxicity was previously reported in another close DEX derivative, ICRF-161 (Martin et al., 2009). Therefore, it was crucial to test the two closest derivatives of DEX prepared in this study (GK-627 and GK580 ) in vivo in a well-established rabbit model of ANT cardiotoxicity to validate our in vitro findings.

Survival and General Toxicity. DAU administered to rabbits in weekly cycles induced unscheduled deaths between the 9th and 11th weeks of the experiment (Fig. 3A). DEX cotreatment completely prevented DAU-induced unscheduled deaths, whereas cotreatment with either GK-627 or GK-580 was unable to provide this protection. Multiple deaths were 


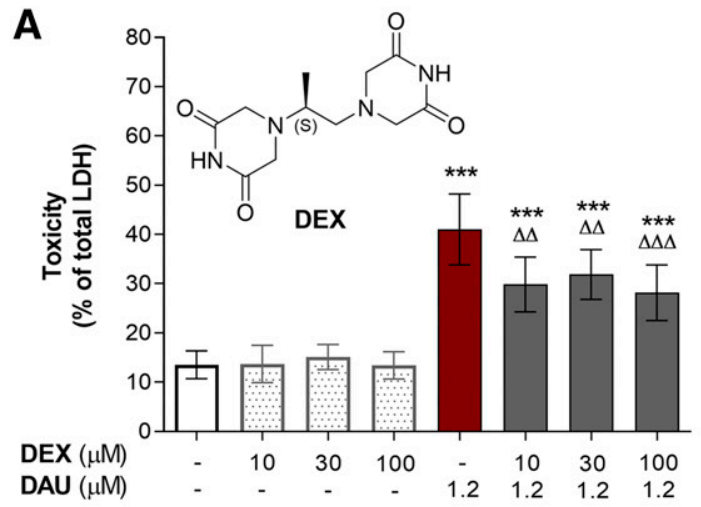

B

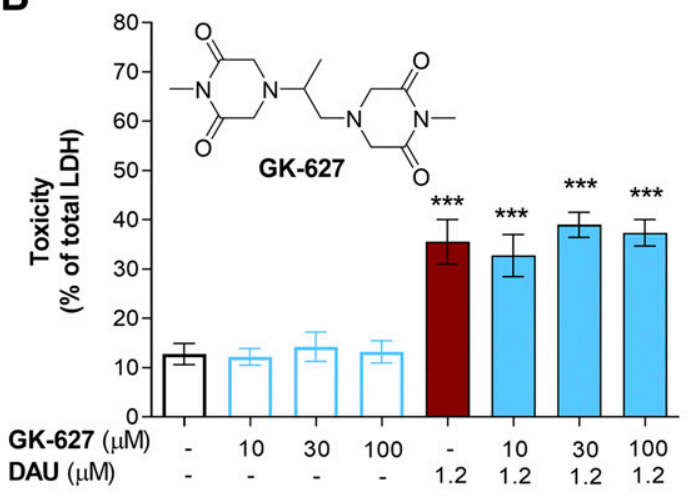

D

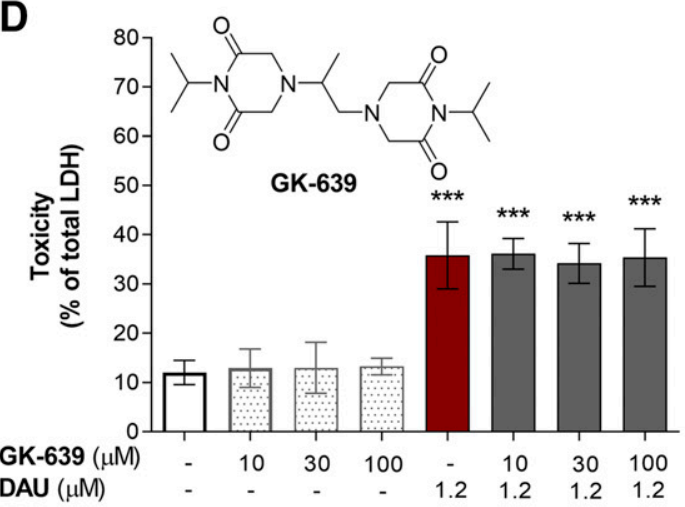

$F$

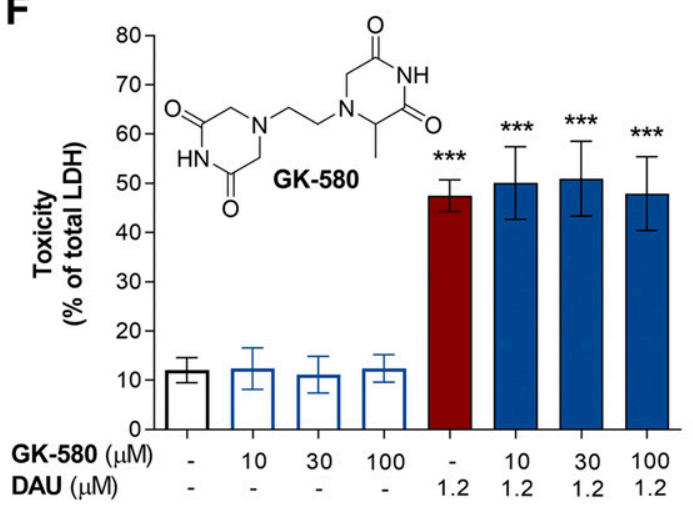

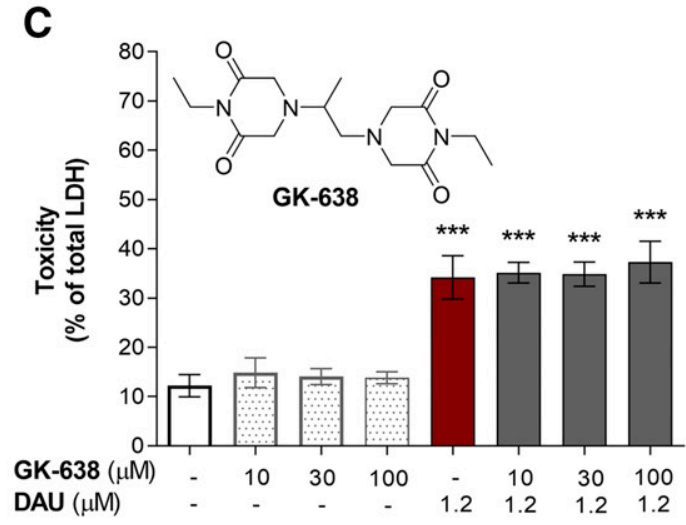
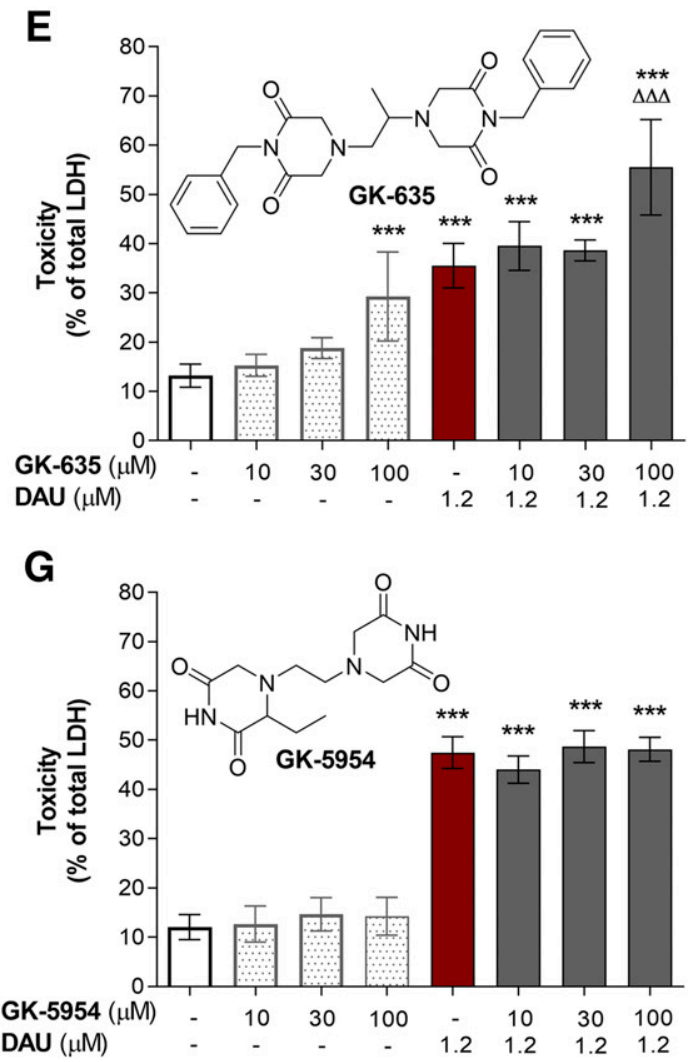

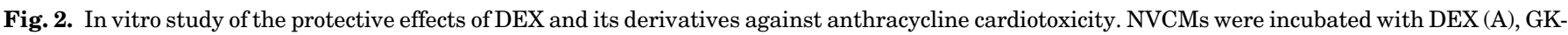

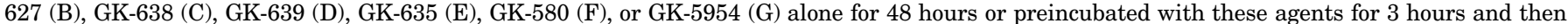

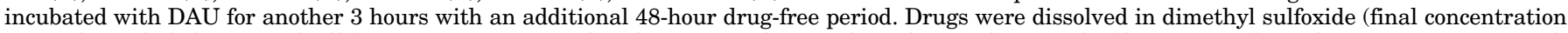

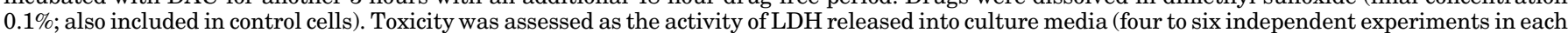

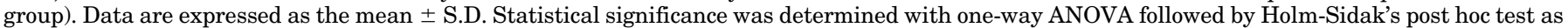
compared with the control group (“***" $P \leq 0.001$ ) and the DAU group (“ $\Delta \triangle " P \leq 0.01 ; " \Delta \triangle \triangle " P \leq 0.001$ ). 
A

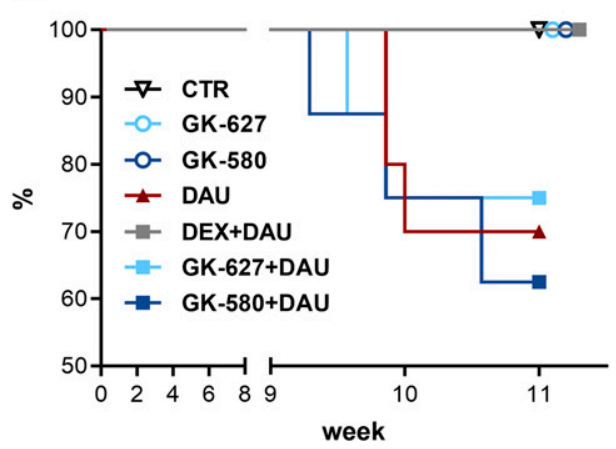

B

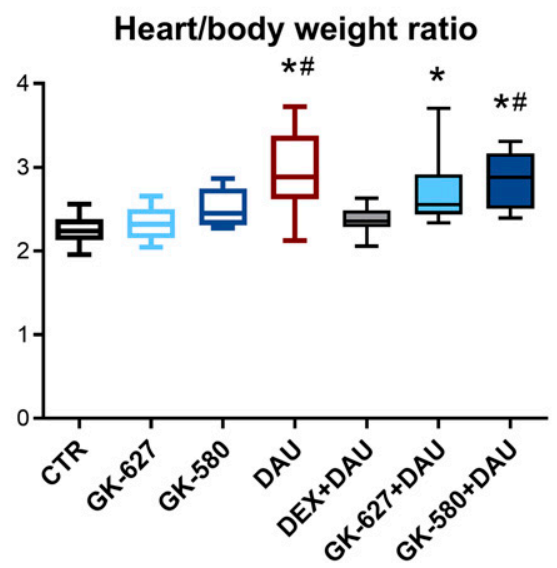

C

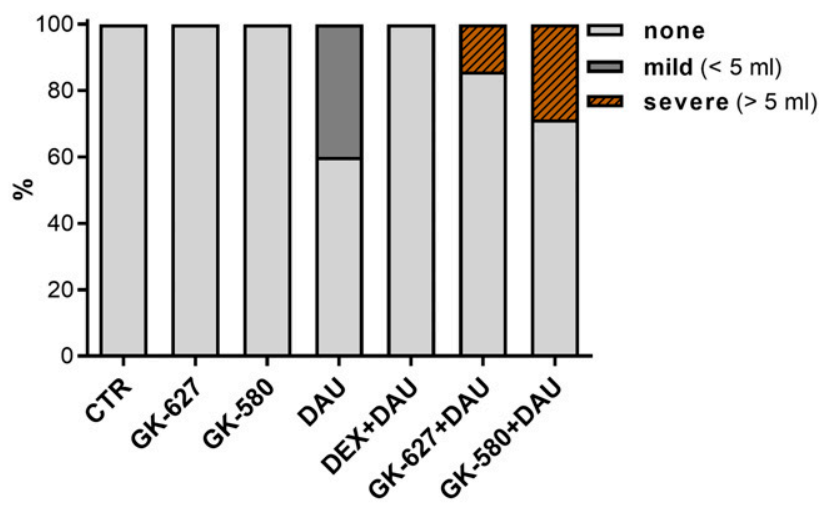

D

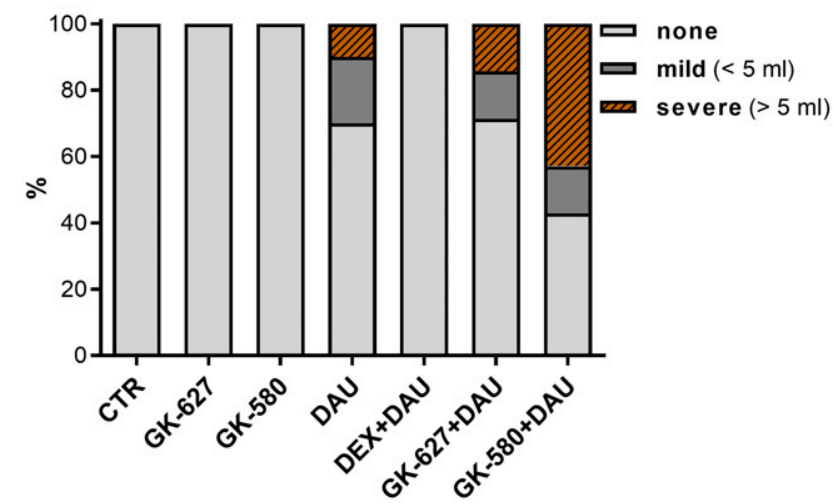

Fig. 3. In vivo study of the protective effects of DEX and its two close derivatives (GK-627 and GK-580) against chronic anthracycline cardiotoxicitysurvival and necropsy findings. Animal survival is expressed in the Kaplan-Meier curve (A). Necropsy findings are displayed as follows: heart/body weight ratio (B), incidence of hydrothorax (C), and ascites (D). Data are expressed as medians with boxes and whiskers representing the interquartile range and 5th-95th percentiles, respectively, (B) or as a percentage of animals with fluid retention in the given range (C and D). Statistical significance was evaluated using one-way ANOVA followed by Holm-Sidak's post hoc test (B). Symbols indicate statistically significant differences $(P<0.05)$ in comparison with the following: “*," CTR group; and "\#," DEX + DAU group. The results from all animals in the study were included in the analysis: CTR, GK-627, GK-627 + DAU, GK-580 + DAU ( $n=8$ in each group), GK-580 $(n=5)$, DAU $(n=10)$, and DEX + DAU $(n=7)$.

observed in the latter groups with timings and extents similar to those in the DAU-alone group. No unscheduled deaths were found in the groups treated with GK-627 or GK580 alone. Necropsy examinations revealed LV or biventricular dilation and a significant rise in the heart/body weight ratio in all animal groups receiving DAU, except for the DEX + DAU group (Fig. 3B). DAU treatment also induced signs of blood congestion in the pleural (hydrothorax; Fig. 3C) and peritoneal cavities (ascites, Fig. 3D). Although DEX clearly prevented both signs of DAUinduced blood congestion, neither of the two close derivatives was able to prove the same effect. No apparent changes in behavior were noted in the animals, with the exception of the tendency to decrease food intake and locomotive activity several days before unscheduled death in DAU-treated animals.

Examination of LV Function. Both echocardiographic and LV catheterization examinations revealed impaired LV systolic function after DAU treatment (Fig. 4, A and B). DEX cotreatment prevented the development of systolic dysfunction, as evident from both examinations. However, neither GK-627 nor GK-580 cotreatment provided any protection. The LV dysfunctions in both of these combination groups were similar to that observed in the DAU group.
Biomarkers of Increased LV Wall Stress and Myocardial Damage. The myocardial gene expressions of the atrial and brain natriuretic peptides as markers of $\mathrm{LV}$ wall stress and heart failure were significantly and markedly increased in all groups receiving DAU compared with those in the control group, except for the DEX + DAU group, in which expressions of both genes were low and insignificant (Fig. 4, C and D). These findings further confirm the results of examinations of LV function presented above and suggest that only DEX prevents DAU-induced LV dysfunction and heart failure.

DAU-induced myocardial damage was also quantitatively documented by a significant increase in the plasma concentration of cTnT at the end of the study (Fig. 4E) as well as by the increased area under the curve (AUC) of plasma concentrations of this biomarker throughout the experiment (Fig. 4F). Although DEX cotreatment evidently prevented the increase of this biomarker, this was not the case for its two derivatives.

Histopathological Examination of the LV Myocardium. Conspicuous morphologic changes characteristic of chronic ANT treatment were found in the hearts of rabbits in the DAU group (Fig. 5). Typical focal damage was manifested by different degrees of cell degeneration; that is, the fragmentation and loss of myofibrils, shrinkage up to disintegration of 
A

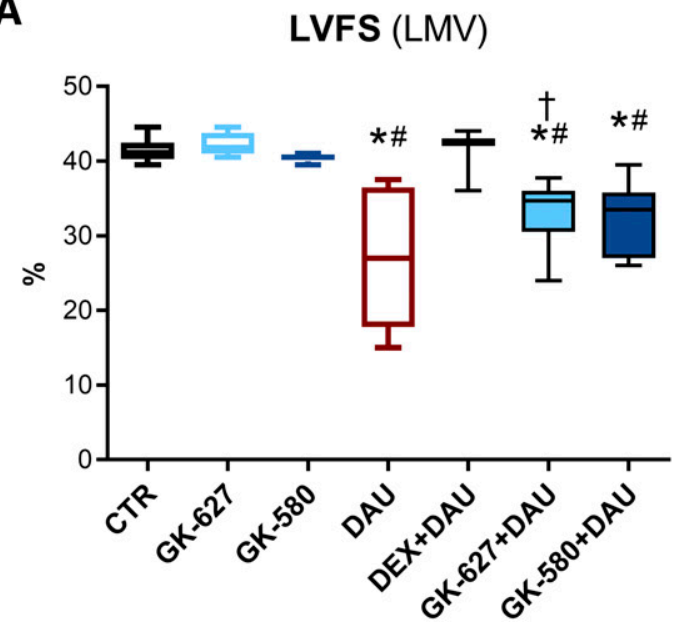

C

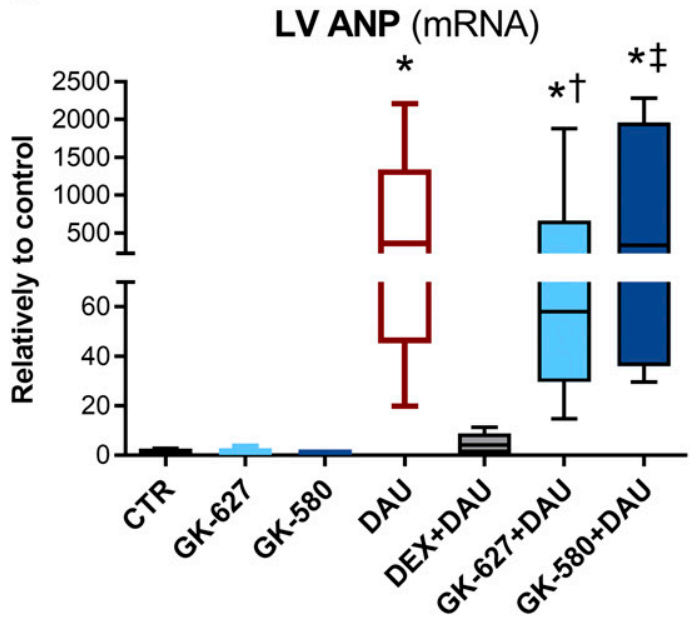

E

Plasma cTnT (LMV)

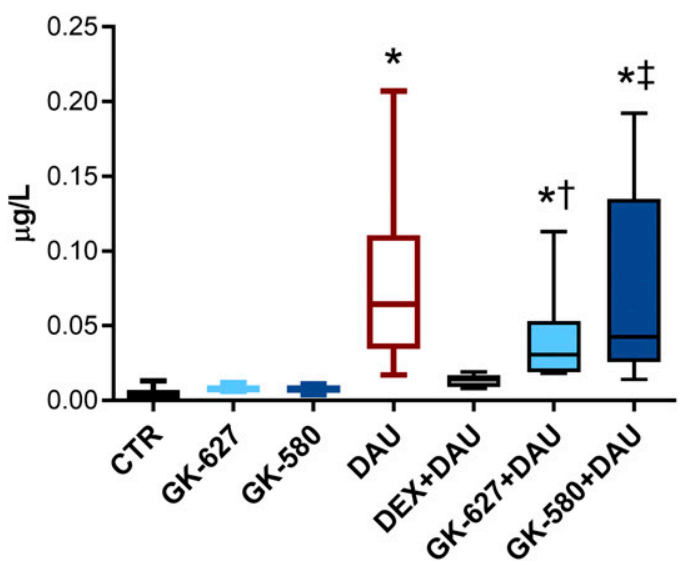

B

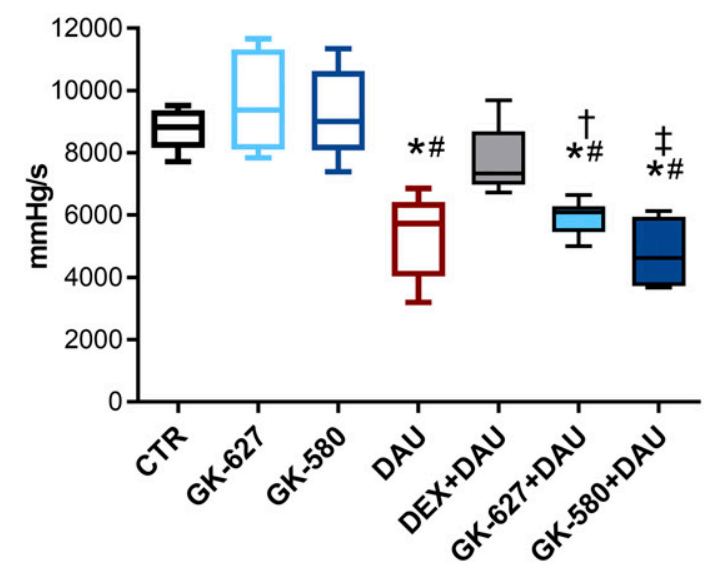

D

LV BNP (mRNA)

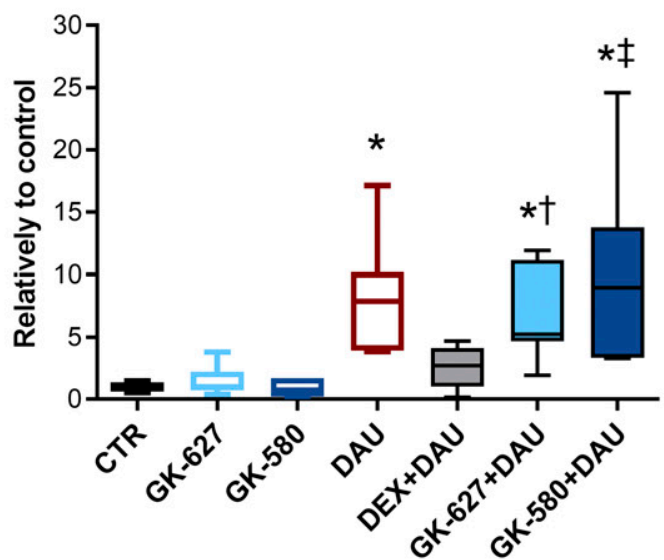

$\mathbf{F}$

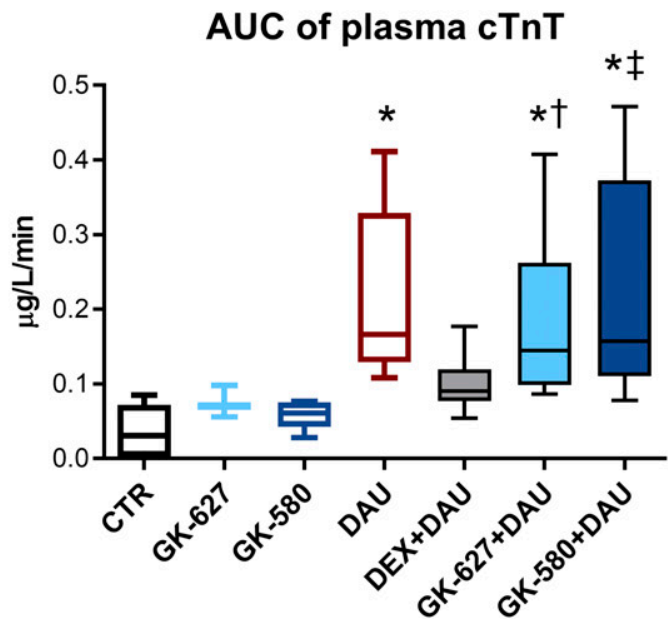

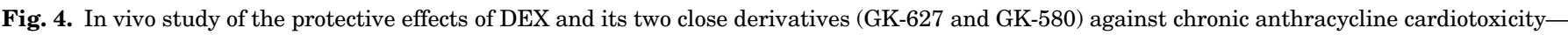

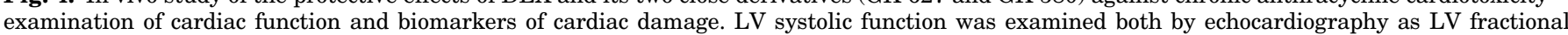

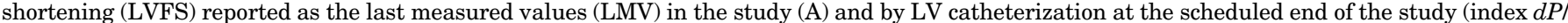

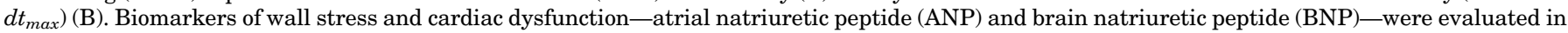

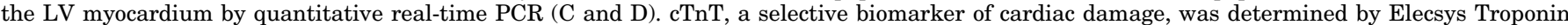

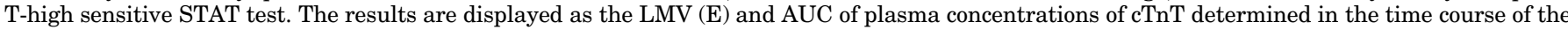

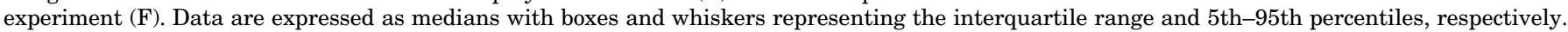

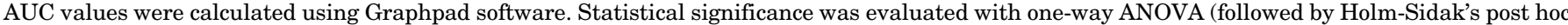

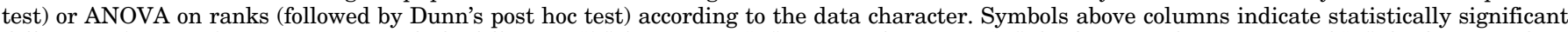

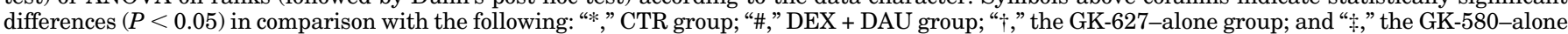




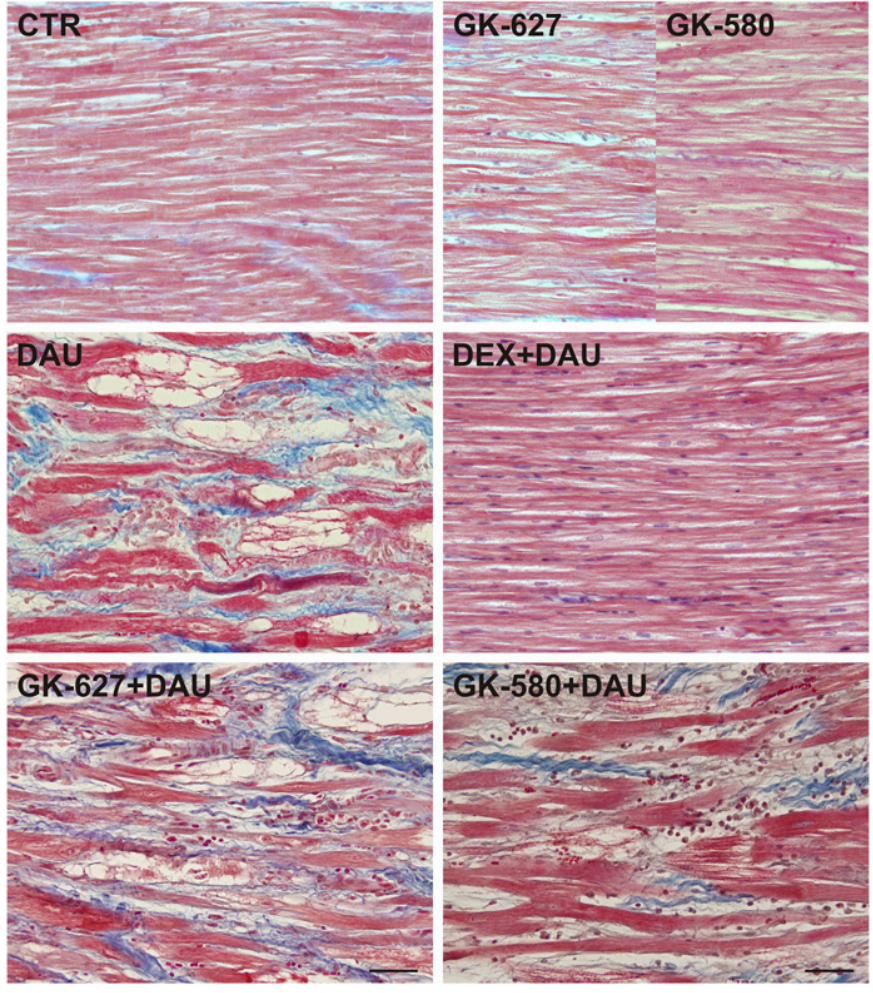

Fig. 5. In vivo study of the protective effects of DEX and its two close derivatives (GK-627 and GK-580) against chronic anthracycline cardiotoxicity-morphologic alterations in the left ventricular myocardium. Normal structure of the myocardium was obvious not only in the CTR, GK627-alone, and GK-580-alone groups, but it was almost normal in the DEX + DAU group. In contrast, conspicuous focal degenerative changes manifesting themselves by the fragmentation of myofibrils (intensely eosinophilic) but mainly by vacuolization of the cytoplasm of cardiomyocytes (with a wide range of vacuole size) scatter groups of macrophages removing cell debris (e.g., in the center of the picture) resulting in replacement fibrosis (dark blue-stained bundles of collagen fibers) were characteristic of the DAU group. In the combination groups with dexrazoxane derivatives (GK-627 + DAU and GK-580 + DAU), the myocardium showed very similar morphologic damage to that in the DAU-alone group. Masson's trichrome staining. Scale bar, $50 \mu \mathrm{m}$.

the cell nucleus, and gradually increasing vacuolization of the cytoplasm, resulting in cell death. Necrotic cardiomyocytes are gradually replaced by fibrotic scar tissue, representing important healing process. The most distinct changes were found in the myocardia of animals with unscheduled death. In contrast, almost normal myocardial structures were found in the hearts of animals in the DEX + DAU group. Degenerating cardiomyocytes were rarely present herein. Hence, the morphologic image was completely different from that of the DAU group. In combination groups with both GK-627 and GK-580, the quality and quantity of myocardial damage were comparable to those in the DAU group. The control, GK-627, and GK-580-alone groups presented normal myocardial structures.

Taken together, both DEX derivatives under study, GK-627 and GK-580, were unable to prevent DAU-induced myocardial damage, which is in accordance with our other in vivo findings.

\section{Pharmacokinetic Study of GK-627 and GK-580 and Comparison with DEX}

All of the experimental evidence presented above conclusively demonstrated that both studied DEX derivatives, GK627 and GK-580, are ineffective as cardioprotectants in vivo when administered at the same doses and through the same route of administration as DEX. However, we could not exclude the fact that all of these in vivo results may be (co) determined by unexpected differences in pharmacokinetics compared with those of DEX. Therefore, we performed a pharmacokinetic experiment to examine whether the failure of these agents to provide cardioprotective effects stems from different pharmacokinetics or pharmacodynamics in comparison with DEX.

In these experiments, DEX and both its derivatives were administered in a single intraperitoneal injection $(60 \mathrm{mg} / \mathrm{kg})$ as in the cardioprotective experiments described above, and the concentrations of the drugs were determined in plasma by LC-MS/MS. The concentration-time profiles of the parent compounds in plasma determined in these experiments are shown in Fig. 6A. Similar to DEX, both DEX derivatives were readily absorbed after intraperitoneal administration, with peak plasma concentrations achieved within the first two sampling intervals (10-20 minutes), that is, before the administration of DAU in the cardioprotective experiments (30 minutes). The peak plasma concentrations $\left(c_{\max }\right)$ were very similar in DEX and GK-627 (324 \pm 102 and $330 \pm 17 \mu \mathrm{M}$, respectively), whereas the same parameter was only moderately lower in GK-580 (204 $\pm 105 \mu \mathrm{M})$. The plasma concentrations of GK-627 were even higher than those of DEX at most time intervals, and the total exposure to this DEX derivative was significantly higher, as confirmed by AUC calculation (Fig. 6C). This largely excludes the hypothesis that the failure of GK-627 as a cardioprotectant is caused by distinct pharmacokinetics of the parent compound compared with those of DEX. In the case of GK-580, the plasma concentrations in the majority of time intervals were moderately lower than those in the DEX group, but the AUC was still very close to that of DEX. Although this could potentially determine minor relative quantitative differences in the cardioprotective effect of this compound, it certainly cannot explain the complete lack of effect in GK-580 in contrast to the robust effect seen in the DEX combination group.

Both compounds were designed to retain the ability to undergo hydrolytic opening of bisdioxopiperazine rings to metal-chelating agents similar to DEX, which has been traditionally considered as a prodrug of the metal-chelating metabolite ADR-925. Hence, we analyzed the plasma concentrations of the metabolites of both DEX derivatives analogical to ADR-925 (Fig. 1). Although the plasma concentrations of the metabolite were even higher in GK-627 than in DEX (Fig. 6B), the opposite was true for GK-580. The same is also valid for the AUC of the metabolites (Fig. 6D). Although there was some difference in metabolism between these two derivatives, the data do not suggest that the impaired ability to form the two ring-opened metabolites could be a universal

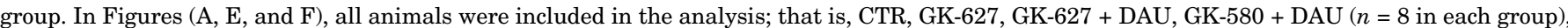

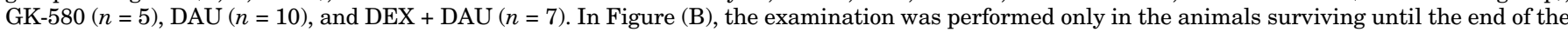

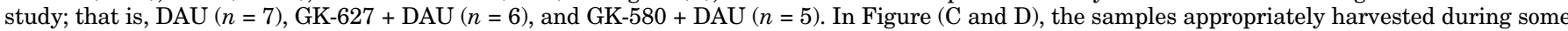
unscheduled deaths of animals were included in the analysis: DAU $(n=10)$, GK-627 + DAU $(n=7)$, and GK-580 + DAU $(n=6)$. 
A

\section{Plasma PK - parent compounds}

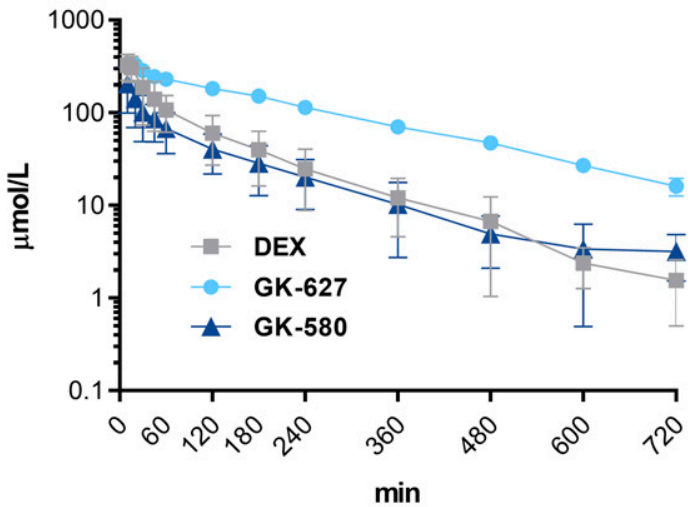

C

\section{AUC of parent compounds}

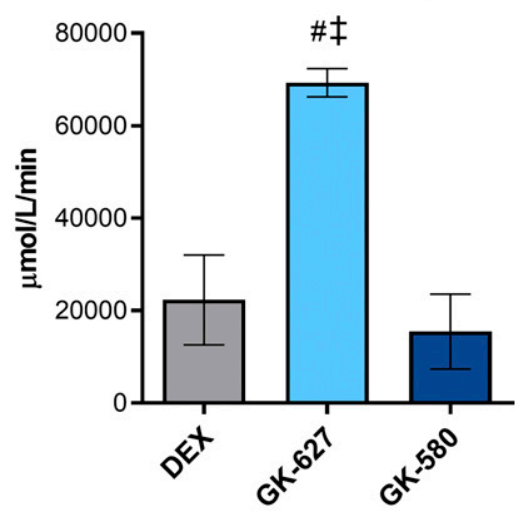

B
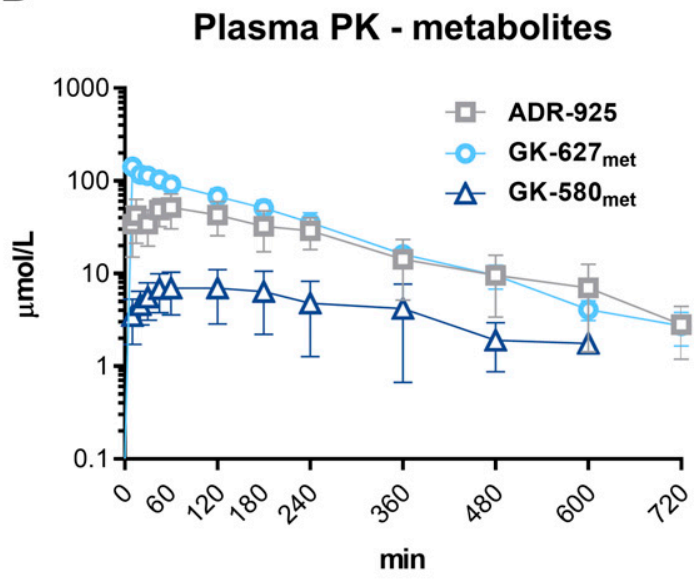

D

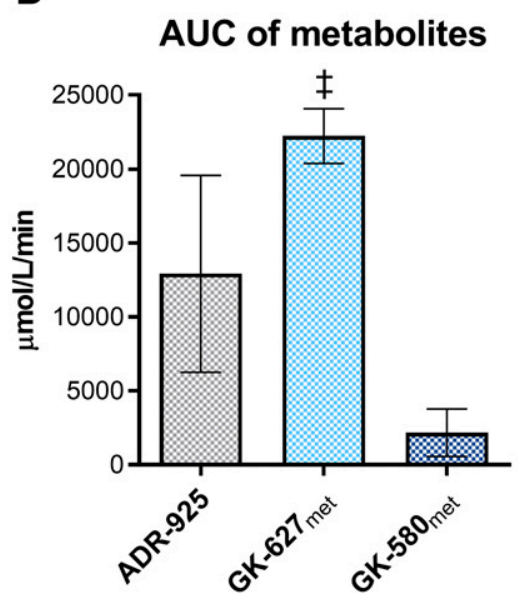

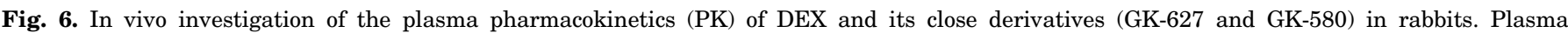

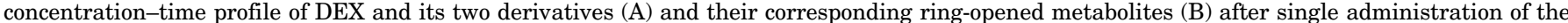

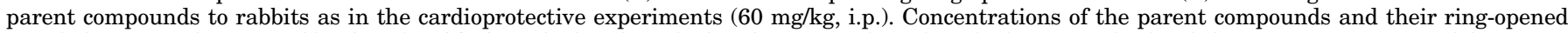

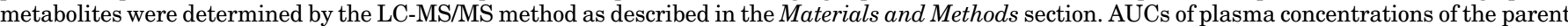

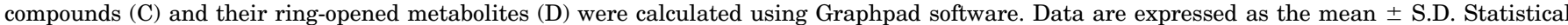

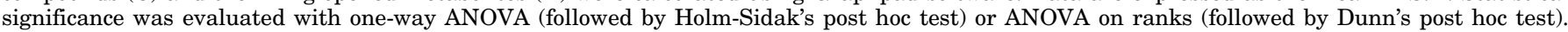

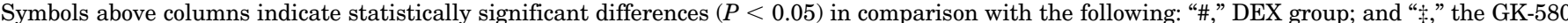

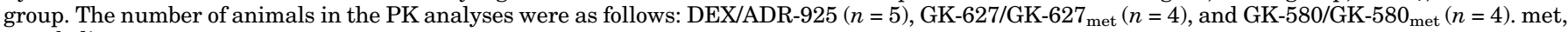
metabolite.

explanation for the lack of cardioprotective effects in the studied DEX derivatives.

\section{Iron-Chelating Properties of DEX Derivatives and Their Metabolites}

Pharmacokinetic study largely excluded that the major reason for the striking difference in cardioprotective effects between DEX and both its derivatives was in their different pharmacokinetic properties. Hence, we focused on the differences in pharmacodynamics. Because the metal-chelating hypothesis of the cardioprotective effects of DEX is still one of the most cited, we checked the chelating capabilities of both DEX derivatives and-mainly - their ring-opened metabolites in the iron displacement assay from complex with DAU. The displacement was determined spectrophotometrically as the decline in absorbance of the DAU-Fe complex at $600 \mathrm{~nm}$. The change in absorbance induced by addition of the tested compound $(100 \mu \mathrm{M})$ for 600 seconds is displayed both as the time course and percentage of control (DAU $+\mathrm{Fe})$ at the end of the experiment (Fig. 7, A and B). A potent iron chelator, salicylaldehyde isonicotinoyl hydrazone and a DEX ringopened metabolite (ADR-925) were used as positive controls. Although both parent compounds (GK-627 and GK-580) were unable to displace iron from the DAU-Fe complex as expected (the results were the same as in the controls), their two ringopened metabolites were chelating iron to the same extent as ADR-925 and to a similar extent to salicylaldehyde isonicotinoyl hydrazone. These results demonstrate that the difference in cardioprotective potential between DEX and its derivatives cannot be explained by the different chelating properties of their metabolites.

\section{Interaction of DEX Derivatives with Top2B and Comparison with DEX}

We next focused on Top2B, which has been considered as an important target for ANT cardiotoxicity (Zhang et al., 2012).

Two types of assays were used to assess the interaction of DEX and its derivatives with Top2B. First, the inhibition of 
A

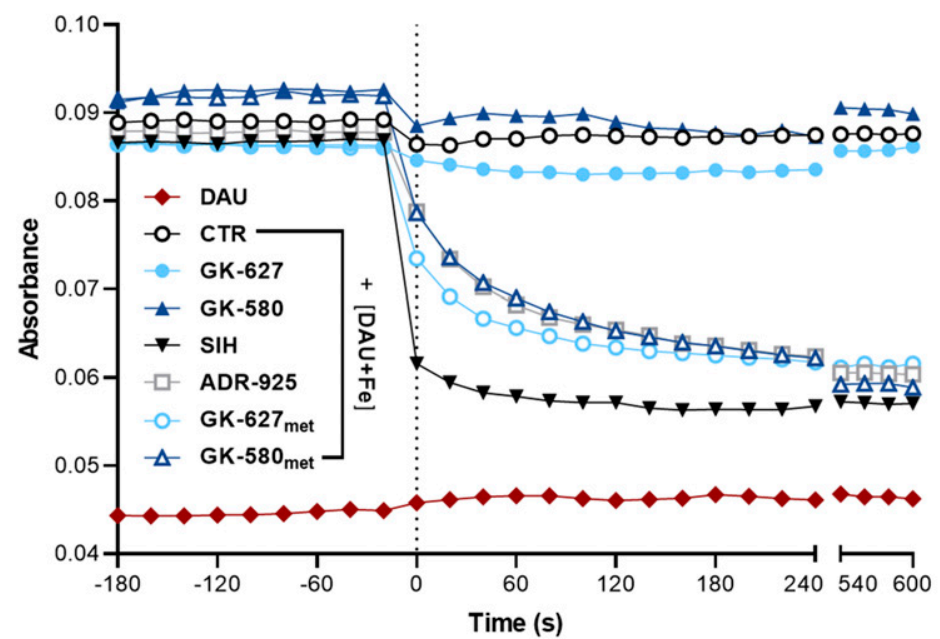

B

Relative Fe displacement at $600 \mathrm{~s}$

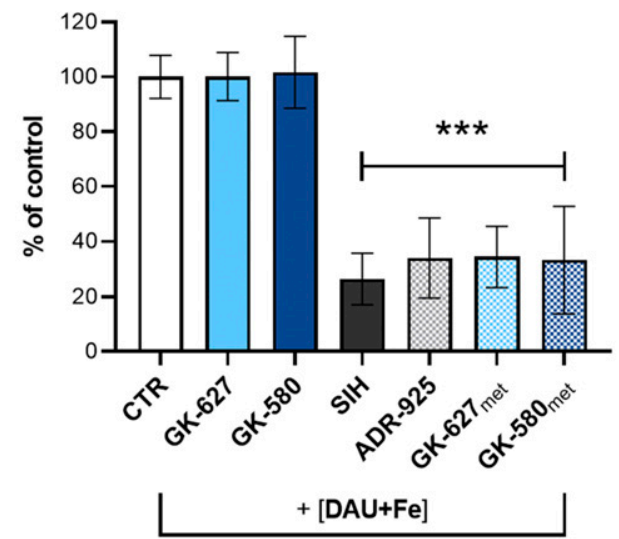

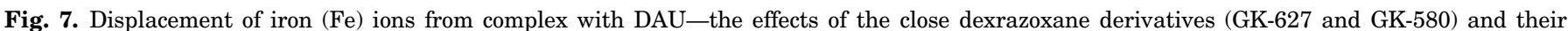

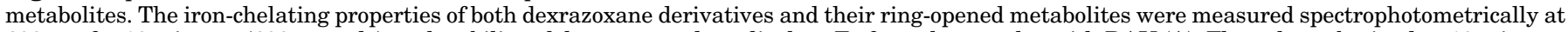

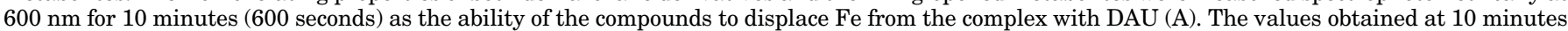

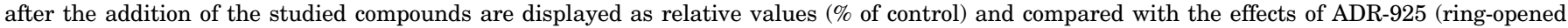

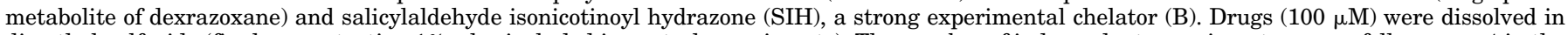

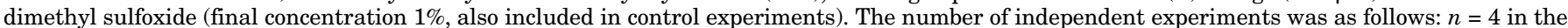

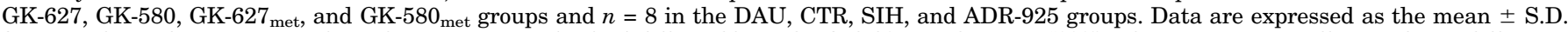

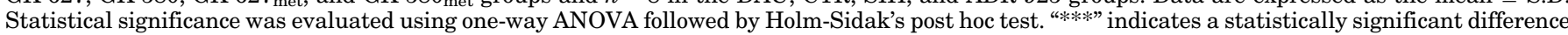
$(P \leq 0.001)$ compared with the control group $(\mathrm{CTR}+\mathrm{DAU}+\mathrm{Fe})$. met, metabolite.

purified human Top2B relaxation activity was evaluated in vitro using supercoiled plasmid DNA. In this setting, only DEX substantially inhibited Top2B, whereas none of its six derivatives had comparable activity in concentrations up to $1 \mathrm{mM}$ (Fig. 8A). Second, depletion of the Top2B enzyme was assessed at the protein level using samples obtained from both in vitro and in vivo experiments with the same setup as that used in the cardioprotective experiments. In vitro, DEX clearly depleted Top2B in NVCMs, whereas none of its derivatives were observed to have comparable activity (Fig. 8B). The same was confirmed for GK-627 and GK-580 in vivo (Fig. 8C). Although DEX significantly depleted Top2B in the LV myocardium of rabbits 12 hours after its administration, neither of the two closest DEX derivatives showed the same effect, although they were present at similar or higher concentrations in the rabbit blood. Hence, the studied modifications of DEX resulted in the loss of the ability to interact with purified human Top2B enzyme, and the same was observed in primary animal cardiomyocytes in vitro and in vivo in animal hearts. This could be a plausible reason for the complete lack of cardioprotective effects in both settings.

\section{Interaction of DEX Derivatives with Top2A and Their Impact on the Antiproliferative Effects of DAU}

Since DEX is also known to inhibit Top2A, which largely explains its potential to inhibit the proliferation of cancer cells, we also tested the studied DEX derivatives for these effects.

Indeed, DEX inhibited the relaxation activity of purified human Top2A enzyme (Supplemental Fig. 1) and dosedependently inhibited proliferation of the leukemic cell line HL-60 (10-100 $\mu \mathrm{M}$, Supplemental Fig. 2A). In contrast, none of the DEX derivatives showed a similar inhibitory effect on Top2A (Supplemental Fig. 1). The effects of all DEX derivatives on the proliferation of HL-60 cells were small and insignificant at concentrations below $10 \mu \mathrm{M}$ (Supplemental Fig. 2A). At higher concentrations (30-100 $\mu \mathrm{M})$, only GK-635 and GK-5954 affected the proliferation of HL-60 cells. Since the former compound was also significantly toxic to nonproliferating cardiomyocytes, the nonspecific cytotoxic effect of this dibenzyl derivative was likely to be involved. Similar results were observed in combinations of DEX or its derivatives $(10$ or $100 \mu \mathrm{M})$ with DAU (Supplemental Fig. 2, B-H). Although DEX significantly enhanced the antiproliferative effects of DAU, its derivatives showed no significant effect in these settings.

\section{Discussion}

In this study, we demonstrated that the simple alkylation of DEX on both imides or in position 3 of one of the dioxopiperazine rings resulted in: 1 ) the complete loss of cytoprotective effects against ANT toxicity in NVCMs in vitro at concentrations up to $100 \mu \mathrm{M}$ and 2) the loss of cardioprotective effects against chronic ANT cardiotoxicity in rabbits in vivo when administered at the same dose as DEX in terms of the prevention of unscheduled deaths, LV systolic dysfunction, signs of blood congestion, biomarkers of heart failure, cardiac damage, and myocardial histopathology. The lack of protective effects in vivo was not due to different pharmacokinetics of the studied derivatives compared with those of DEX. Instead, the principal difference was most likely in the pharmacodynamics and particularly in the ability to interact with Top2B, which highlights this protein as a key target for the induction of effective cardioprotection.

An important finding of this study is that the cardioprotective effects of DEX show a very tight SAR because even simple methylation at the given positions completely abolished its cardioprotective potential in the studied range of concentrations 


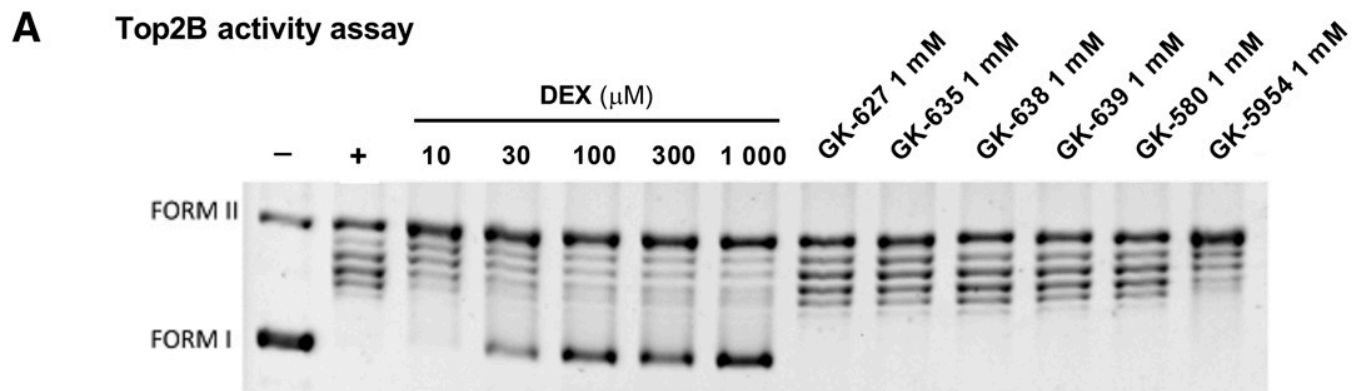

B Top2B depletion in NVCMs
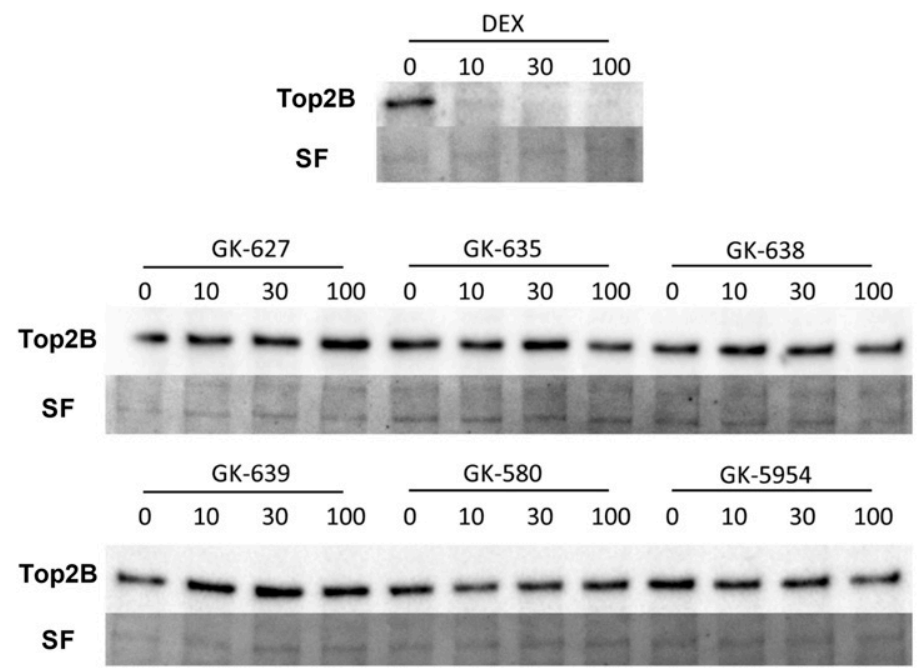

C

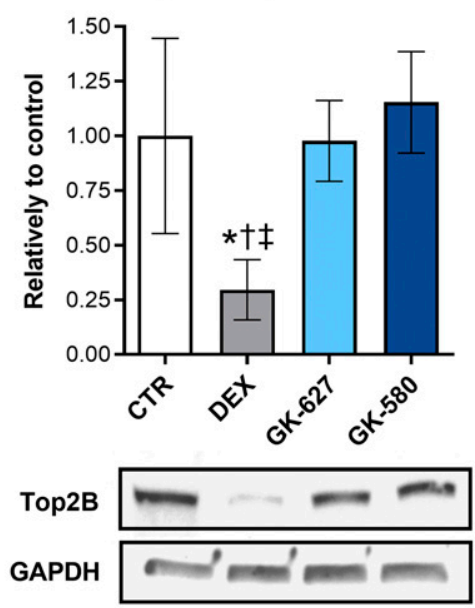

Fig. 8. Effects of DEX and its close derivatives on Top2B activity and its protein abundance in vitro and in vivo. Inhibition of purified human Top2B by DEX and its derivatives (A); lanes: "-" supercoiled TCS1 plasmid (substrate) without Top2B, “+” plasmid relaxed by Top2B in the presence of $1 \%$ dimethyl sulfoxide. DEX concentration dependently inhibited Top2B activity, whereas DEX derivatives had no effect. Top2B protein abundance in NVCMs (B) and rabbit LV myocardium (C) after treatment with DEX or its derivatives was assayed by Western blotting. In vitro, DEX and its derivatives were used at concentrations of $0-100 \mu \mathrm{M}$. In vivo DEX, GK-627, and GK-580 were administered at $60 \mathrm{mg} / \mathrm{kg}$, i.p., and the myocardium was taken for analysis 12 hours later. DEX depleted Top2B protein (mol. mass $\approx 183 \mathrm{kD}$ ) in both the NVCMs and LV myocardium, whereas the DEX derivatives showed no effects. Protein loading was controlled by analysis of the total protein [Stain-Free (SF)] (B) or glyceraldehyde-3-phosphate dehydrogenase $(\mathrm{GAPDH})(\mathrm{mol}$. mass $\approx 37 \mathrm{kD})(\mathrm{C})$. Results are displayed as representative analyses $(\mathrm{A}$ and $\mathrm{B})$ or as mean $\pm \mathrm{S} . \mathrm{D}$. (C), in which the number of animals was as follows: $n=5$ in the CTR and DEX groups, and $n=4$ in the GK-627 and GK-580 groups. Statistical significance was evaluated using one-way ANOVA (followed by Holm-Sidak's post hoc test). Symbols indicate statistically significant differences $(P<0.05)$ compared with the following: “*” CTR group; “†,” the GK-627 group; and “†,” the GK-580 group.

and doses. Although the substitution of the dioxopiperazine ring was addressed in this study for the first time, the functionalization of both imides with methyl has been previously studied. Herman et al. (1985) observed minimal protective activity of the dimethyl DEX derivative ICRF-239 (our GK627) against the massive overall toxicity of an acute high dose of doxorubicin in hamsters, as judged by a simple survival analysis. However, no conclusion can be drawn from these experiments regarding the cardioprotective potential of the studied agents. More relevant data were reported later by Herman et al. (1997): ICRF-239 (our GK-627) decreased the myocardial lesion score in a DEX-validated model of chronic ANT cardiotoxicity in spontaneously hypertensive rats, although this effect was less effective than that observed in DEX. In contrast, our in vitro and in vivo data conclusively showed that neither of the parameters of ANT cardiotoxicity was significantly improved by this DEX derivative, despite achieving even higher plasma concentrations than those after DEX administration. Notably, in the former study, no animal survived until the end of the experiment in the group cotreated with this derivative, which was in contrast with the complete prevention of mortality induced by DEX administration. This certainly complicated the interpretation of the cardioprotective potential of this compound in a previous study. The incidence of unscheduled deaths in our study was consistent with the lack of cardioprotective potential of the compound.

We cannot exclude that the observed results may be caused by a relative decrease in the potency of these new derivatives compared with that of DEX; however, such difference would have to be a major one. The in vitro results indicate at least 10fold and 33-fold lower potencies than that of DEX in cytoprotective and Top2B inhibitory effects, respectively. Although we do not have the data to estimate the difference in potency in vivo, a relatively marked difference is also expected. No sign of cardioprotection was observed with the studied derivatives at the upper limit of recommended doses for $\operatorname{DEX}(20: 1$ ratio to ANT) despite even higher total body exposures of GK-627. Dose-escalation studies with these agents have not been performed because of the assumed limited additional value and uncertain feasibility of these experiments. 
The current understanding of the SAR of bisdioxopiperazines in cardioprotective settings may be summarized as follows. The presence of unsubstituted imide nitrogens in bisdioxopiperazine is required for the cardioprotective effects of DEX and its related compounds. This, however, concerns only permanent substitutions of this moiety. Morpholinomethyl derivatives (such as ADR-559) or sobuzoxane, which are readily hydrolysable in biologic environments to freeimide bisdioxopiperazines, have been shown to be protective against ANT cardiotoxicity (Herman et al., 1997; Vavrova et al., 2013). Data from the present study suggest the same requirement for position 3 on the 2,6-dioxopiperazine rings, as its alkylation practically abolished the cardioprotective effects. We have previously found that oxo groups of dioxopiperazine rings are also essential for cardioprotective effects (Jirkovská-Vávrová et al., 2015), and our unpublished results suggest that the removal of only a single oxo group resulted in the loss of protective effects in vitro (unpublished data). Others reported that the two-carbon linker between the rings is required for cardioprotection against chronic ANT cardiotoxicity because one-carbon elongation results in a lack of cardioprotective effects in vivo despite comparable pharmacokinetics to DEX (Martin et al., 2009). Herman et al. (1997) showed that a demethylated DEX derivative (ICRF-154) was cardioprotective, suggesting that the methyl on the linker between the dioxopiperazine rings was not necessary for cardioprotection. However, the poor solubility of this symmetric derivative precludes the exploitation of its pharmacological potential. Our previous in vitro findings with sobuzoxane, which is a prodrug of ICRF-154, confirms this hypothesis. These observations also imply that it is the presence of the methyl group on one of the dioxopiperazine rings that precludes the cardioprotective effect of the isomethyl derivative GK-580 and not the corresponding lack of this substituent on the aliphatic linker. Interestingly, apart from ICRF-154 and DEX (and their prodrugs), neither DEX derivatives studied so far have conclusively shown cardioprotective effects against ANT cardiotoxicity in vivo.

Regarding the mechanism of the cardioprotective effects of DEX and its derivatives, our study strongly indicates that the interaction of DEX with Top2B is essential and indispensable for its cardioprotective effects both in vitro and in vivo. Details of this requirement are poorly understood; it is still not clear whether Top2B inhibition is sufficient for this effect or whether depletion is also a mandatory part of the effect. However, the lack of Top2B interaction predicted the absence of cardioprotective potential in all derivatives tested both in this study and in our previous study (Jirkovská-Vávrová et al., 2015). Previously, Martin et al. (2009) reported a co-incidence of the absence of Top2 inhibitory and in vivo cardioprotective effects against chronic ANT cardiotoxicity in another close DEX derivative (ICRF-161). Because Top2B is the predominant Top2 isoform in cardiomyocytes (Vejpongsa and Yeh, 2014; Atwal et al., 2019), this finding fits the above-mentioned mechanistic concept. Indeed, Top2B has been implicated in the development of ANT cardiotoxicity (Zhang et al., 2012) and in DEX-induced cardioprotection (Lyu et al., 2007; Deng et al., 2014; Lenčová-Popelová et al., 2016). Hence, data from this study contribute to accumulating evidence that the interaction of bisdioxopiperazines with this target is indispensable for effective cardioprotection.
In the current literature, it is often stated that ANT cardiotoxicity may be mediated by both Top2B and metalcatalyzed ROS production, whereas DEX is suggested to prevent both these events (Henninger and Fritz, 2017; Hutchins et al., 2017). However, the findings of this study do not support this hypothesis. At least some of the DEX derivatives evaluated in this study are evidently effectively metabolized to metal-chelating metabolites, which can displace iron from its complexes with DAU, similar to the DEX metabolite ADR-925. However, despite these retained metalchelating effects, no signs of even partial protection were observed in these derivatives lacking Top2B inhibitory effects. We have previously shown that a different DEX derivative [JR-311; (Bures et al., 2017)], which is very prone to hydrolysis of its dioxopiperazine rings to the metal-chelating metabolite, failed to provide effective protection in vitro unless a sufficient amount of the parent compound (allowing effective interaction with Top2B) was not provided through repeated dosing throughout the study. Hence, our data seem to be in accordance with previous reports suggesting that the metalchelating effects of DEX may not be the most important factor for its cardioprotective effects (Minotti et al., 1999). It is noteworthy that some older reports have questioned the physiologic relevance of the ANT-Fe complexes (3:1), which should be targeted by ADR-925 to prevent ROS formation (Gelvan and Samuni, 1988). However, further studies specifically designed to directly address the role of the metalchelating metabolites of DEX in cardioprotection are needed to resolve this question.

In conclusion, this study revealed the very tight SAR of bisdioxopiperazines in cardioprotective settings. It has been previously reported that the dimethyl derivative of DEX retains certain cardioprotective potential, but this study conclusively shows the opposite. For the first time it has been shown that even the smallest substitution with aliphatic alkyls on imides or in position 3 on one of the 2,6dioxopiperazine rings practically abolishes the cardioprotective effects of DEX both in vitro and in vivo. Pharmacokinetic studies confirmed that the reasons for a lack of cardioprotective effects are not in the pharmacokinetics of new derivatives but rather in the pharmacodynamics and the lack of Top2B interaction. These findings further support the notion that Top2B is an upstream druggable target for effective cardioprotection against ANT cardiotoxicity. The complex in vivo part of this study confirmed the predictive value and validated our in vitro models. In bisdioxopiperazine derivatives, a focus on the substitution of the aliphatic linker may be more promising than modifications to the dioxopiperazine rings.

\section{Acknowledgments}

The authors thank Klára Lindrová and Jitka Pohorská for their excellent laboratory assistance.

\section{Authorship Contributions}

Participated in research design: Kollárová-Brázdová, Jirkovská, Štěrbová-Kovaříková, Roh, Šimůnek, Štěrba.

Conducted experiments: Kollárová-Brázdová, Jirkovská, Karabanovich, Pokorná, Bavlovič Piskáčková, Jirkovský, Kubeš, LenčováPopelová, Mazurová, Skalická, Štěrbová-Kovaříková, Roh, Štěrba.

Contributed new reagents or analytic tools: Karabanovich, Bavlovič Piskáčková, Adamcová, Štěrbová-Kovaříková, Roh. 
Performed data analysis: Kollárová-Brázdová, Jirkovská, Karabanovich, Bavlovič Piskáčková, Kubeš, Lenčová-Popelová, Mazurová, Adamcová, Štěrbová-Kovaříková, Roh, Štěrba.

Wrote or contributed to the writing of the manuscript: KollárováBrázdová, Jirkovská, Karabanovich, Štěrbová-Kovaříková, Roh, Šimůnek, Štěrba.

\section{References}

Atwal M, Swan RL, Rowe C, Lee KC, Lee DC, Armstrong L, Cowell IG, and Austin CA (2019) Intercalating TOP2 poisons attenuate topoisomerase action at higher concentrations. Mol Pharmacol 96:475-484.

Bloom MW, Hamo CE, Cardinale D, Ky B, Nohria A, Baer L, Skopicki H, Lenihan DJ, Gheorghiade M, Lyon AR, et al. (2016) Cancer therapy-related cardiac dysfunction and heart failure: part 1: definitions, pathophysiology, risk factors, and imaging. Circ Heart Fail 9:e002661.

Bures J, Jirkovska A, Sestak V, Jansova H, Karabanovich G, Roh J, Sterba M, Simunek T, and Kovarikova P (2017) Investigation of novel dexrazoxane analogue JR-311 shows significant cardioprotective effects through topoisomerase IIbeta but not its iron chelating metabolite. Toxicology 392:1-10.

Creighton AM (1976) inventor, National Research Development Corporation, assignee. Bis diketopiperazines. Patent US3941790A. 1976 Mar 2

Creighton AM (1992) inventor, National Research Development Corporation, assignee. Cardioprotective bis-dioxopiperazines. Patent GB2245832A 1992 Jan 15.

Creighton AM, Hellmann K, and Whitecross S (1969) Antitumour activity in a series of bisdiketopiperazines. Nature 222:384-385.

Deng S, Yan T, Jendrny C, Nemecek A, Vincetic M, Gödtel-Armbrust U, and Wojnowski L (2014) Dexrazoxane may prevent doxorubicin-induced DNA damage via depleting both topoisomerase II isoforms. BMC Cancer 14:842.

EMC (2019) Daunorubicin $20 \mathrm{mg}$ Powder for I.V. Injection - Summary of Product Characteristics (SmPC) [online], https://www.medicines.org.uk/emc/product/4004/ smpc [Accessed 20 Feb. 2020].

Gelvan D and Samuni A (1988) Reappraisal of the association between adriamycin and iron. Cancer Res 48:5645-5649.

Gilroy KL and Austin CA (2011) The impact of the C-terminal domain on the interaction of human DNA topoisomerase II $\alpha$ and $\beta$ with DNA. PLoS One 6:e14693.

Hasinoff BB (1989) The interaction of the cardioprotective agent ICRF-187 [+)-1,2-bis(3,5dioxopiperazinyl-1-yL)propane); its hydrolysis product (ICRF-198); and other chelating agents with the $\mathrm{Fe}(\mathrm{III})$ and $\mathrm{Cu}(\mathrm{II})$ complexes of adriamycin. Agents Actions 26:378-385.

Hasinoff BB, Hellmann K, Herman EH, and Ferrans VJ (1998) Chemical, biological and clinical aspects of dexrazoxane and other bisdioxopiperazines. Curr Med Chem 5:1-28.

Hasinoff BB, Kuschak TI, Yalowich JC, and Creighton AM (1995) A QSAR study comparing the cytotoxicity and DNA topoisomerase II inhibitory effects of bisdioxopiperazine analogs of ICRF-187 (dexrazoxane). Biochem Pharmacol 50 953-958

Hasinoff BB, Patel D, and Wu X (2003) The oral iron chelator ICL670A (deferasirox) does not protect myocytes against doxorubicin. Free Radic Biol Med 35:1469-1479.

Hellmann K (1993) inventor, WINDLESHAW ENTERPRISES LTD, assignee. Bis(3,5-dioxoalkylpiperazine) derivatives for use in preventing or treating conditions that involve iron metabolism. Patent WO9310104A1. 1993 May 27.

Henninger C and Fritz G (2017) Statins in anthracycline-induced cardiotoxicity: rac and Rho, and the heartbreakers. Cell Death Dis 8:e2564.

Herman EH, el-Hage AN, Creighton AM, Witiak DT, and Ferrans VJ (1985) Comparison of the protective effect of ICRF-187 and structurally related analogues against acute daunorubicin toxicity in Syrian golden hamsters. Res Commun Chem Pathol Pharmacol 48:39-55.

Herman EH, Hasinoff BB, Steiner R, and Lipshultz SE (2014) A review of the preclinical development of dexrazoxane. Prog Pediatr Cardiol 36:33-38

Herman EH, Zhang J, Hasinoff BB, Chadwick DP, Clark JR Jr, and Ferrans VJ (1997) Comparison of the protective effects against chronic doxorubicin cardiotoxicity and the rates of iron (III) displacement reactions of ICRF-187 and other bisdiketopiperazines. Cancer Chemother Pharmacol 40:400-408.

Hutchins KK, Siddeek H, Franco VI, and Lipshultz SE (2017) Prevention of cardiotoxicity among survivors of childhood cancer. Br J Clin Pharmacol 83:455-465.

Jirkovská-Vávrová A, Roh J, Lenčová-Popelová O, Jirkovský E, Hrušková K Potǔčková-Macková E, Jansová H, Hašková P, Martinková P, and Eisner T (2015) Synthesis and analysis of novel analogues of dexrazoxane and its open-ring hydrolysis product for protection against anthracycline cardiotoxicity in vitro and in vivo. Toxicol Res (Camb) 4:1098-1114.

Jirkovský E, Lenčová-Popelová O, Hroch M, Adamcová M, Mazurová Y, Vávrová J, Mičuda S, Šimůnek T, Geršl V, and Stěrba M (2013) Early and delayed cardioprotective intervention with dexrazoxane each show different potential for prevention of chronic anthracycline cardiotoxicity in rabbits. Toxicology 311:191-204.

Kim H, Kang HJ, Park KD, Koh KN, Im HJ, Seo Jj, Lee JW, Chung NG, Cho B, Kim HK, et al. (2019) Risk factor analysis for secondary malignancy in dexrazoxanetreated pediatric cancer patients. Cancer Res Treat 51:357-367.
Kovarikova P, Pasakova-Vrbatova I, Vavrova A, Stariat J, Klimes J, and Simunek T (2013) Development of LC-MS/MS method for the simultaneous analysis of the cardioprotective drug dexrazoxane and its metabolite ADR-925 in isolated cardiomyocytes and cell culture medium. J Pharm Biomed Anal 76:243-251.

Lee MP, Sander M, and Hsieh TS (1989) Single strand DNA cleavage reaction of duplex DNA by Drosophila topoisomerase II. J Biol Chem 264:13510-13518.

Lenčová-Popelová $\mathrm{O}$, Jirkovský $\mathrm{E}$, Jansová $\mathrm{H}$, Jirkovská-Vávrová A, VostatkováTichotová L, Mazurová Y, Adamcová M, Chládek J, Hroch M, Pokorná Z, et al. (2016) Cardioprotective effects of inorganic nitrate/nitrite in chronic anthracycline cardiotoxicity: comparison with dexrazoxane. J Mol Cell Cardiol 91:92-103.

Liang C-M, Turner NA, and Witiak DT (1999) inventors, Wisconsin Alumni Research Foundation, assignee. Treatment of vascular leakage and related syndrome such as septic shock by administration of metalloproteinase inhibitors. Patent US5866570A. 1999 Feb 2.

Lyu YL, Kerrigan JE, Lin C-P, Azarova AM, Tsai Y-C, Ban Y, and Liu LF (2007) Topoisomerase IIbeta mediated DNA double-strand breaks: implications in doxorubicin cardiotoxicity and prevention by dexrazoxane. Cancer Res 67:8839-8846.

Martin E, Thougaard AV, Grauslund M, Jensen PB, Bjorkling F, Hasinoff BB, Tjørnelund J, Sehested M, and Jensen LH (2009) Evaluation of the topoisomerase II-inactive bisdioxopiperazine ICRF-161 as a protectant against doxorubicininduced cardiomyopathy. Toxicology 255:72-79.

Minotti G, Cairo G, and Monti E (1999) Role of iron in anthracycline cardiotoxicity: new tunes for an old song? FASEB J 13:199-212.

Paul C, Liliemark J, Tidefelt U, Gahrton G, and Peterson C (1989) Pharmacokinetics of daunorubicin and doxorubicin in plasma and leukemic cells from patients with acute nonlymphoblastic leukemia. Ther Drug Monit 11:140-148.

Pfaffl MW (2001) A new mathematical model for relative quantification in real-time RT-PCR. Nucleic Acids Res 29:e45.

Popelová O, Štěrba M, Hasková P, Simůnek T, Hroch M, Guncová I, Nachtigal P, Adamcová M, Geršl V, and Mazurová Y (2009) Dexrazoxane-afforded protection against chronic anthracycline cardiotoxicity in vivo: effective rescue of cardiomyocytes from apoptotic cell death. Br J Cancer 101:792-802.

Reichardt P, Tabone M-D, Mora J, Morland B, and Jones RL (2018) Risk-benefit of dexrazoxane for preventing anthracycline-related cardiotoxicity: re-evaluating the European labeling. Future Oncol 14:2663-2676.

Simůnek T, Klimtová I, Kaplanová J, Mazurová Y, Adamcová M, Štěrba M, Hrdina $\mathrm{R}$, and Geršl V (2004) Rabbit model for in vivo study of anthracycline-induced heart failure and for the evaluation of protective agents. Eur J Heart Fail 6:377-387.

Simůnek T, Stérba M, Popelová O, Adamcová M, Hrdina R, and Geršl V (2009) Anthracycline-induced cardiotoxicity: overview of studies examining the roles of oxidative stress and free cellular iron. Pharmacol Rep 61:154-171.

Stěrba M, Popelová $\mathrm{O}$, Vávrová $\mathrm{A}$, Jirkovský E, Kovaříková $\mathrm{P}$, Geršl V, and Simůnek $\mathrm{T}$ (2013) Oxidative stress, redox signaling, and metal chelation in anthracycline cardiotoxicity and pharmacological cardioprotection. Antioxid Redox Signal 18: 899-929

Swain SM, Whaley FS, Gerber MC, Weisberg S, York M, Spicer D, Jones SE, Wadler S, Desai A, Vogel C, et al. (1997) Cardioprotection with dexrazoxane for doxorubicin-containing therapy in advanced breast cancer. J Clin Oncol 15: 1318-1332

Swamy KCK, Kumar NNB, Balaraman E, and Kumar KVPP (2009) Mitsunobu and related reactions: advances and applications. Chem Rev 109:2551-2651.

Tebbi CK, London WB, Friedman D, Villaluna D, De Alarcon PA, Constine LS, Mendenhall NP, Sposto R, Chauvenet A, and Schwartz CL (2007) Dexrazoxaneassociated risk for acute myeloid leukemia/myelodysplastic syndrome and other secondary malignancies in pediatric Hodgkin's disease. J Clin Oncol 25:493-500.

van Dalen EC, Caron HN, Dickinson HO, and Kremer LCM (2011) Cardioprotective interventions for cancer patients receiving anthracyclines. Cochrane Database Syst Rev (6):CD003917.

Varatharajan S, Panetta JC, Abraham A, Karathedath S, Mohanan E, Lakshmi KM, Arthur N, Srivastava VM, Nemani S, George B, et al. (2016) Population pharmacokinetics of Daunorubicin in adult patients with acute myeloid leukemia. Cancer Chemother Pharmacol 78:1051-1058.

Vavrova A, Jansova H, Mackova E, Machacek M, Haskova P, Tichotova L, Sterba M, and Simunek T (2013) Catalytic inhibitors of topoisomerase II differently modulate the toxicity of anthracyclines in cardiac and cancer cells. PLoS One 8:e76676.

Vejpongsa P and Yeh ETH (2014) Topoisomerase 2 $\beta$ : a promising molecular target for primary prevention of anthracycline-induced cardiotoxicity. Clin Pharmacol Ther 95:45-52.

Zhang S, Liu X, Bawa-Khalfe T, Lu L-S, Lyu YL, Liu LF, and Yeh ET (2012) Identification of the molecular basis of doxorubicin-induced cardiotoxicity. Nat Med 18: 1639-1642.

Address correspondence to: Dr. Martin Štěrba, Department of Pharmacology, Faculty of Medicine in Hradec Králové, Charles University, Simkova 870, Hradec Králové, 500 03, Czech Republic. E-mail: sterbam@lfhk.cuni.cz 